\title{
GROUP QUASI-REPRESENTATIONS AND INDEX THEORY
}

\author{
MARIUS DADARLAT
}

\begin{abstract}
Let $M$ be a closed connected manifold and let $D$ be an elliptic operator on $M$. Let $G$ be a discrete countable group and let $\widetilde{M} \rightarrow M$ be a principal $G$-bundle. Connes and Moscovici showed that this data defines an analytic index $\operatorname{ind}_{\ell^{1}(G)}(D) \in K_{0}\left(\ell^{1}(G)\right)$. If $B$ is a unital tracial $\mathrm{C}^{*}$-algebra, we give a formula for the trace of the image of ind $_{\ell^{1}(G)}(D)$ in $K_{0}(B)$ under the map induced by a quasi-representation of $G$ in $B$. As an application, we reprove and generalize a formula of Exel and Loring to surface groups.
\end{abstract}

\section{INTRODUCTION}

Let $M$ be a closed connected Riemannian manifold with fundamental group $G$ and let $D$ be an elliptic operator on $M$. Connes, Gromov and Moscovici [5] introduced the notion of almost flat bundle and proved an index theorem showing that the pushforward of the equivariant index of $D$ under a quasi-representation of $G$ coming from parallel transport in an almost flat bundle $E$ on $M$ is equal to the index of $D$ twisted by $E$. They used this result to show that the signature with coefficients in an almost flat bundle is a homotopy invariant.

In this paper we take a dual approach where the input data involves a group quasi-representation rather than an almost flat bundle. In conjunction with a finite dimensional approximation technique based on quasidiagonality, this approach enables us to use the classic Atiyah-Singer index theorem [1] and respectively the Mishchenko-Fomenko index theorem [20] to give a new proof and a generalization of the index theorem of Connes, Gromov and Moscovici that allows for almost flat bundles $E$ with fibers projective Hilbert modules over a tracial $\mathrm{C}^{*}$-algebra, see Theorem 3.6. In the last section, we reprove and generalize the Exel-Loring formula [9] to surface groups, by reinterpreting it as an index theorem, see Theorem 4.2.

2010 Mathematics Subject Classification. 46L35, 46L80, 19 K35.

The author was partially supported by NSF grant \#DMS-1101305. 
Let $G$ be a discrete countable group, let $\mathcal{G} \subset G$ be a finite set and let $\omega>0$. Let $B$ be a unital $\mathrm{C}^{*}$-algebra. A map $\pi: G \rightarrow B$ is called a $(\mathcal{G}, \omega)$-unitary representation if it satisfies the following conditions:

(i) $\pi(1)=1,\|\pi(s)\| \leq 1$ for all $s \in G$.

(ii) $\left\|\pi\left(s^{-1}\right)-\pi(s)^{*}\right\|<\omega$ for all $s \in \mathcal{G}$,

(iii) $\|\pi(s t)-\pi(s) \pi(t)\|<\omega$ for all $s, t \in \mathcal{G}$.

Let $A$ be a unital involutive Banach algebra, let $\mathcal{G} \subset A$ be a finite set and let $\omega>0$. A unital linear contraction $\pi: A \rightarrow B$ such that $\left\|\pi\left(a^{*}\right)-\pi(a)^{*}\right\|<\omega$ and $\left\|\pi\left(a a^{\prime}\right)-\pi(a) \pi\left(a^{\prime}\right)\right\|<\omega$ for all $a, a^{\prime} \in \mathcal{G}$ is called a $(\mathcal{G}, \omega)$-*-representation of $A$ in $B$. We will use the term quasirepresentation to refer to a $(\mathcal{G}, \omega)$-unitary representation or to a $(\mathcal{G}, \omega)$-*representation where $\mathcal{G}$ and $\omega$ are not necessarily specified. Let us note that a quasi-representation of $G$ induces a quasi-representation of the involutive Banach algebra $\ell^{1}(G)$ by the formula: $\widehat{\pi}\left(\sum_{s \in G} \lambda_{s} \cdot s\right)=\sum_{s \in G} \lambda_{s} \pi(s)$. Moreover, given a finite set $\widehat{\mathcal{G}} \subset \ell^{1}(G)$ and $\widehat{\omega}>0$ there exist a finite set $\mathcal{G} \subset G$ and $\omega>0$ such that if $\pi: G \rightarrow B$ is a $(\mathcal{G}, \omega)$-unitary representation, then $\widehat{\pi}: \ell^{1}(G) \rightarrow B$ is a $(\widehat{\mathcal{G}}, \widehat{\omega})-*$-representation. An important method for turning K-theoretical invariants of $G$ into numerical invariants is to use quasi-representations $\pi: G \rightarrow M_{k}(\mathbb{C})$ to pushforward idempotents in matrices over $\ell^{1}(G)$ or $C^{*}(G)$ to scalar idempotents [5]. It is then very natural to seek quasi-representations that act non-trivially on K-theory. This question is addressed in [7] where we study the existence of sequences of quasi-representations $\pi_{n}: C^{*}(G) \rightarrow M_{k(n)}(\mathbb{C})$ that induce prescribed maps $K_{0}\left(C^{*}(G)\right) \rightarrow \mathbb{Z}$ and we give conditions for $G$ under which all the K-theory classes in $K^{0}(B G)$ are almost flat. The first example of a topologically nontrivial quasi-representation is due to Voiculescu [27] for $G=\mathbb{Z}^{2}$.

Suppose that $M$ is a closed smooth connected manifold. Let $G$ be a discrete countable group and let $\widetilde{M} \rightarrow M$ be a principal $G$-bundle. The diagonal action of $G$ on $\widetilde{M} \times \ell^{1}(G)$ defines a flat $\ell^{1}(G)$-bundle $\widetilde{M} \times{ }_{G} \ell^{1}(G) \rightarrow M$ which gives an element $\ell \in K_{0}\left(C(M) \otimes \ell^{1}(G)\right)$ (Mishchenko's line bundle). Using Lafforgue's cap product (see [16]) $K K^{\text {ban }}\left(\mathbb{C}, C(M) \otimes \ell^{1}(G)\right) \times$ $K K^{\text {ban }}(C(M), \mathbb{C}) \rightarrow K K^{\text {ban }}\left(\mathbb{C}, \ell^{1}(G)\right),(y, z) \mapsto y \circ(z \otimes 1)$, one defines a $\operatorname{map} \mu: K_{0}(M) \rightarrow K_{0}\left(\ell^{1}(G)\right)$, by $\mu(z)=\ell \circ(z \otimes 1)$. For Banach algebras $A$, $B$ we denote by $A \otimes B$ the completion of the algebraic tensor product with respect to the maximal Banach norm such that $\|a \otimes b\| \leq\|a\|\|b\|$.

The goal of the paper is to provide a formula for the push-forward of $\mu(z)$, $z \in K_{0}(M)$, under a quasi-representation $\pi$ of $\ell^{1}(G)$. To make this precise, we write $\mu(z)=\left[e_{0}\right]-\left[e_{1}\right]$ where $e_{0}, e_{1}$ are idempotents in $M_{m}(\mathbb{C}) \otimes \ell^{1}(G)$. If $\pi: \ell^{1}(G) \rightarrow M_{k}(\mathbb{C})$ is sufficiently multiplicative (this depends on the 
choice of the representatives $\left.e_{0}, e_{1}\right)$, then $\left(i d_{m} \otimes \pi\right)\left(e_{i}\right)$ can be perturbed canonically to idempotents $f_{i}$ in $M_{m k}(\mathbb{C}), i=0,1$. We are interested in computing the integer $\pi_{\sharp}(\mu(z)):=\left[f_{0}\right]-\left[f_{1}\right] \in K_{0}(\mathbb{C})=\mathbb{Z}$. More generally, if $\pi: \ell^{1}(G) \rightarrow B$ is a sufficiently multiplicative quasi-representation in a unital $\mathrm{C}^{*}$-algebra $B$ and $\tau$ is a tracial state of $B$, we would like to compute $\tau\left(\pi_{\sharp}(\mu(z))\right)$ where $\pi_{\sharp}(\mu(z))$ is defined similarly. We reserve the notation $\mu_{\ell^{1}}$ : $K_{0}(M) \rightarrow K_{0}\left(\ell^{1}(G)\right)$ for a related map defined in [16]. Its definition involves a projection $p \in \ell^{1}\left(G, C_{0}(\widetilde{M})\right)$ (constructed from a cutoff function) and the descent map $j_{G}: K K_{G}^{b a n}\left(C_{0}(\widetilde{M}), \mathbb{C}\right) \rightarrow K K^{b a n}\left(\ell^{1}\left(G, C_{0}(\widetilde{M})\right), \ell^{1}(G)\right)$. The author does not know if $\mu_{\ell^{1}}=\mu$ in this general context. If $\widetilde{M} \rightarrow M$ is the universal cover of $M$, then $\mu_{\ell^{1}}$ is the Lafforgue's $\ell^{1}$-version of the assembly map and it is known that the images of $\mu_{\ell^{1}}(z)$ and $\mu(z)$ coincide under the map $K_{0}\left(\ell^{1}(G)\right) \rightarrow K_{0}\left(C^{*}(G)\right)$, see [16]. We will verify that if $\mathcal{G}$ is sufficiently large and $\omega$ is sufficiently small, then for any $(\mathcal{G}, \omega)$-unitary representation $\pi: G \rightarrow B, \pi_{\sharp}\left(\mu_{\ell^{1}}(z)\right)=\pi_{\sharp}(\mu(z))$, see Corollary 3.5. The computation $\pi_{\sharp}\left(\mu_{\ell^{1}}(z)\right)$ is given by Theorem 3.6. This is obtained by combining a technical result, Theorem 3.2 (see also Corollary 3.5), with the MishchenkoFomenko index theorem [20], [24]. The case of a matricial $\mathrm{C}^{*}$-algebra $B=M_{k}(\mathbb{C})$ yields a result that is closely related to the index theorem of Connes, Gromov and Moscovici [5]. Our approach, which is very different from [5], is based on descriptions of the isomorphism $K K^{\text {ban }}(\mathbb{C}, A) \cong K_{0}(A)$ and of the cap product $K K^{\text {ban }}(\mathbb{C}, A \otimes B) \times K K^{\text {ban }}(A, \mathbb{C}) \rightarrow K K^{\text {ban }}(\mathbb{C}, B)$ that involve compressions by quasi-central idempotents, see Corollary 2.4 and Proposition 2.5. In the last part of the paper we bring a new perspective and generalize the Exel-Loring formula [9] to surface groups by realizing it as an index formula.

The author would like to thank Walther Paravacini for useful discussions on $K K^{b a n}$-theory and to the referee for his thorough suggestions and criticism that lead to an improved exposition.

\section{IDEMPOTENTS IN BANACH ALGEBRAS AND $K K^{\text {ban }}$-THEORY}

In this section we study various perturbation properties of idempotents in a Banach algebra. Proposition 2.5 is a key tool for the rest of the paper, for it allows us to approach index computations via compressions by quasi-central idempotents. We work with Lafforgue's $K K^{\text {ban }}$-theory [16] in a simplified version which does require the use of dual pairs. This equivalent approach is used in the survey [15]. A written account of why the two approaches are equivalent is given in [22]. 
We begin by a short review of the setup from [15]. Let $A$ be a unital Banach algebra. A right Banach $A$-module is a Banach space $E$ endowed with a right action of $A$ such that $x \cdot 1=x$ and $\|x a\| \leq\|x\|\|a\|$ for all $x \in E$ and $a \in A$. A left Banach $A$-module $F$ is defined similarly. We denote by $E \otimes_{A} F$ the completion of $E \otimes^{\text {alg }} F$ under the largest seminorm such that $\|x \otimes a y-x a \otimes y\|=0$ and $\|x \otimes y\| \leq\|x\|\|y\|$, for all $x \in E, y \in F, a \in A$.

Let $E$ and $F$ be right Banach $A$-modules. The space of $\mathbb{C}$-linear continuous maps $T: E \rightarrow F$ such that $T(x a)=T(x) a$ for $x \in E$ and $a \in A$ is denoted by $L_{A}(E, F)$ or $L_{A}(E)$ if $E=F$. The ideal $K_{A}(E)$ of $A$-compact operators is defined as the closure of the linear span of the $A$-rank one operators, i.e. those of the form $S \circ T$ where $S \in L_{A}(A, E)$ and $T \in L_{A}(E, A)$. A Fredholm module over $A$ consists of a $\mathbb{Z} / 2$-graded right Banach $A$-module $E$ and an odd operator $T \in L_{A}(E)$ such that $T^{2}-i d_{E} \in K_{A}(E)$. If $(E, T)$ is a Fredholm module over $A$ and $\theta: A \rightarrow B$ is a unital morphism then $\left(E \otimes_{A} B, T \otimes 1\right)$ is a Fredholm module over $B$. Let $A[0,1]$ be the Banach algebra of continuous functions from $[0,1]$ to $A$ endowed with norm $\|f\|=\sup _{t \in[0,1]}\|f(t)\|_{A}$. Let $\theta_{0}, \theta_{1}: A[0,1] \rightarrow A$ be the evaluations at 0 and 1. Two Fredholm modules over $A$ are homotopic if they are the images under $\theta_{0}$ and $\theta_{1}$ of a Fredholm module over $A[0,1]$. Let us recall that for any unital Banach algebra $A$ there is a functorial bijection between $K_{0}(A)$ and the set of homotopy classes of Fredholm modules over $A$, see [16, Thm. 1.2.8]. $E^{b a n}(A, B)$ is the set of isomorphism classes of triples $(E, \pi, T)$, where $E$ is a $\mathbb{Z} / 2$-graded Banach right $B$-module, $\pi: A \rightarrow L_{B}(E)$ is a morphism of Banach algebras that takes values in even operators, and $T \in L_{B}(E)$ is an odd operator that satisfies $\pi(a)\left(T^{2}-i d_{E}\right) \in K_{B}(E)$ and $\pi(a) T-T \pi(a) \in K_{B}(E)$ for any $a \in A$. One defines $K K^{b a n}(A, B)$ as the set of homotopy classes in $E^{b a n}(A, B)$, where the homotopy relation is defined using $E^{b a n}(A, B[0,1])$. If we are in a situation when $\alpha \in K K^{b a n}(A, B)$ is given by the class of a triplet

$$
\left(H \oplus H, \pi=\left(\begin{array}{cc}
\pi_{0} & 0 \\
0 & \pi_{1}
\end{array}\right), T=\left(\begin{array}{ll}
0 & 1 \\
1 & 0
\end{array}\right)\right),
$$

where $H$ is a right Banach $B$-module, then we will also write $\alpha=\left[\pi_{0}, \pi_{1}\right]$. Here $\pi_{i}: A \rightarrow L_{B}(H)$ have the property that $\pi_{0}(a)-\pi_{1}(a) \in K_{B}(H)$, for all $a \in A$. In the case $A=\mathbb{C}$, we will also write $\alpha=\left[p_{0}, p_{1}\right]$ where $p_{i}$ is the idempotent $p_{i}=\pi_{i}(1)$ and $p_{0}-p_{1} \in K_{B}(H)$. While a general intersection product is not available in $K K^{\text {ban }}$, there are cap products

$$
K_{0}(A) \times K K^{b a n}(A, B) \rightarrow K_{0}(B), \quad(x, z) \mapsto x \circ z,
$$




$$
K_{0}(A \otimes D) \times K K^{b a n}(A, B) \rightarrow K_{0}(B \otimes D) \quad(x, z) \mapsto x \circ(z \otimes 1) .
$$

The product (3) is defined by combining the product (2) with the morphism $K K^{\text {ban }}(A, B) \rightarrow K K^{\text {ban }}(A \otimes D, B \otimes D)$. Recall that $E^{b a n}(\mathbb{C}, B)$ is the set of isomorphism classes of Fredholm modules over $B$ and $K K^{\text {ban }}(\mathbb{C}, B) \cong$ $K_{0}(B)$. With this in mind, assuming that $A$ is unital, one can describe the product (2) as follows (see [15]). If $p$ is an idempotent in $A$, and $(E, \pi, T) \in E^{b a n}(A, B)$, then the product of $[p] \in K_{0}(A)$ with $\alpha=[E, \pi, T] \in$ $K K^{b a n}(A, B)$ is the class of the Fredholm module over $B$ equal to

$$
(\operatorname{Im} \pi(p), \pi(p) T \pi(p)) .
$$

When $p$ is an idempotent in $M_{k}(A)$, we use the image of $p$ by $i d_{k} \otimes \pi$ : $M_{k}(A) \rightarrow L_{B}\left(E^{k}\right)$. If $\alpha$ is of the form (1), then the class of (4) is equal to

$$
\left[\pi_{0}(p), \pi_{1}(p)\right],
$$

since both $\pi(p)(\pi(p) T \pi(p)-T)$ and $(\pi(p) T \pi(p)-T) \pi(p)$ are in $K_{B}(H \oplus H)$. Given $0<\varepsilon \leq 1 / 2$ we consider the following neighborhood of $\{0,1\}$ :

$$
V(\varepsilon)=\{\lambda \in \mathbb{C}:|\lambda|<\varepsilon\} \cup\{\lambda \in \mathbb{C}:|\lambda-1|<\varepsilon\} .
$$

Let $B$ be a unital Banach algebra. Let $x \in B$ and suppose that its spectrum $S p(x)$ is contained in $V(\varepsilon)$. Then we denote by $\chi(x)$ the idempotent

$$
\chi(x)=\frac{1}{2 \pi i} \int_{C}(\lambda-x)^{-1} d z
$$

where $C=\{\lambda \in \mathbb{C}:|\lambda-1|=1 / 2\}$. Note that $\chi(x)$ is well-defined whenever $\left\|x^{2}-x\right\|<1 / 4$. Indeed, suppose that $\left\|x^{2}-x\right\|<\varepsilon^{2}$. If $\lambda \in S p(x)$, then $\lambda^{2}-\lambda \in S p\left(x^{2}-x\right)$ and so $\left|\lambda^{2}-\lambda\right| \leq\left\|x^{2}-x\right\|<\varepsilon^{2}$. It follows that $\lambda \in V(\varepsilon)$.

Definition 2.1. Let $\pi: A \rightarrow B$ be a unital linear contraction of Banach algebras. Let $p \in M_{m}(\mathbb{C}) \otimes A$ be an idempotent and let $x=\left(i d_{m} \otimes \pi\right)(p) \in$ $M_{m}(\mathbb{C}) \otimes B$. We define $\pi_{\sharp}(p)=[\chi(x)] \in K_{0}(B)$ whenever $\left\|x^{2}-x\right\|<1 / 4$ and set $\pi_{\sharp}(p)=0$ otherwise.

One can fix for each K-theory element $\alpha \in K_{0}(A)$, two idempotents $e_{\alpha}, f_{\alpha}$ such that $\alpha=\left[e_{\alpha}\right]-\left[f_{\alpha}\right]$ and define $\pi_{\sharp}: K_{0}(A) \rightarrow K_{0}(B)$ by $\pi_{\sharp}(\alpha)=$ $\pi_{\sharp}\left(e_{\alpha}\right)-\pi_{\sharp}\left(f_{\alpha}\right)$. While this definition of $\pi_{\sharp}$ on $K_{0}(A)$ depends on the choices we make for $e_{\alpha}, f_{\alpha}$, the ambiguity can be eliminated by passing to maps that are more and more multiplicative. Specifically, if $\alpha=\left[e_{\alpha}^{\prime}\right]-\left[f_{\alpha}^{\prime}\right]$ for another set of idempotents $e_{\alpha}^{\prime}, f_{\alpha}^{\prime}$, then $\pi_{\sharp}\left(e_{\alpha}\right)-\pi_{\sharp}\left(f_{\alpha}\right)=\pi_{\sharp}\left(e_{\alpha}^{\prime}\right)-\pi_{\sharp}\left(f_{\alpha}^{\prime}\right)$ if $\pi: A \rightarrow B$ is a unital linear contraction such that $\left\|\pi\left(a a^{\prime}\right)-\pi(a) \pi\left(a^{\prime}\right)\right\|<\omega$ for all $a, a^{\prime}$ in a sufficiently large finite set $\mathcal{G}$ and sufficiently small $\omega$. 
Lemma 2.2. Let $B$ be a unital Banach algebra and let $E$ be a Banach right $B$-module. Let $\left(e_{0}, f_{0}\right)$ and $\left(e_{1}, f_{1}\right)$ be pairs of idempotents in $L_{B}(E)$ such that $e_{0}-f_{0} \in K_{B}(E)$ and $e_{1}-f_{1} \in K_{B}(E)$. If there is $M \geq 0$ such that $\left\|e_{i}\right\| \leq M,\left\|f_{i}\right\| \leq M, i=0,1,\left\|e_{0}-e_{1}\right\|<1 /(8 M+4)$ and $\left\|f_{0}-f_{1}\right\|<1 /(8 M+4)$, then $\left[e_{0}, f_{0}\right]=\left[e_{1}, f_{1}\right]$ in $K K^{\text {ban }}(\mathbb{C}, B) \cong K_{0}(B)$.

Proof. Let $\varepsilon=1 /(8 M+4)$. Define $x, y \in C\left([0,1], L_{B}(E)\right)$ by $x(t)=(1-$ t) $e_{0}+t e_{1}$ and $y(t)=(1-t) f_{0}+t f_{1}$. Then $\left\|x-e_{0}\right\| \leq\left\|e_{1}-e_{0}\right\|<\varepsilon$ and $\left\|y-f_{0}\right\| \leq\left\|f_{1}-f_{0}\right\|<\varepsilon$. Moreover

$$
\left\|x^{2}-x\right\| \leq\|x\|\left\|x-e_{0}\right\|+\left\|x-e_{0}\right\|\left\|e_{0}\right\|+\left\|e_{0}-x\right\|<(2 M+1) \varepsilon \leq 1 / 4 .
$$

Similarly one checks that $\left\|y^{2}-y\right\|<1 / 4$. As observed earlier, it follows that $S p(x)$ and $S p(y)$ are contained in $V(1 / 2)$. Then $e:=\chi(x)$ and $f:=\chi(y)$ are idempotents in $L_{B[0,1]}(E[0,1])$ such that $e-f \in K_{B[0,1]}(E[0,1])$ since $x-y \in K_{B[0,1]}(E[0,1])$ and the Riesz calculus is functorial. It follows that the pair $(e, f)$ gives a homotopy between $\left(e_{0}, f_{0}\right)$ and $\left(e_{1}, f_{1}\right)$.

Lemma 2.3. For any $M \geq 0$ and $0<\varepsilon<1 / 2$ there is $\delta=\delta(M, \varepsilon)>0$ with the following property. If $B$ is a unital Banach algebra and $x, y \in B$ satisfy $\|x\| \leq M,\left\|x^{2}-x\right\| \leq \delta,\|x-y\| \leq \delta$, then $S p(x) \cup S p(y) \subset V(\varepsilon)$ and $\|\chi(y)-x\|<\varepsilon,\|\chi(x)-x\|<\varepsilon,\|\chi(y)-y\|<\varepsilon,\|\chi(x)-\chi(y)\|<\varepsilon$.

Proof. Given $0<\varepsilon<1 / 2$, suppose that $\delta>0$ is sufficiently small so that $2 M \delta+\delta^{2}+2 \delta<\varepsilon^{2}$. Then $\left\|x^{2}-x\right\| \leq \delta<\varepsilon^{2}<1 / 4$ and $\left\|y^{2}-y\right\| \leq$ $\|y\|\|y-x\|+\|y-x\|\|x\|+\left\|x^{2}-x\right\|+\|x-y\| \leq 2 M \delta+\delta^{2}+2 \delta<\varepsilon^{2}<1 / 4$. As observed earlier, from these estimates it follows that $S p(x), S p(y) \subset V(\varepsilon)$. Seeking a contradiction, suppose now that there are $M \geq 0$ and $0<\varepsilon<1 / 2$ for which the lemma is false. It follows that we can find a decreasing sequence $\left(\delta_{n}\right)$ converging to 0 , with $2 M \delta_{n}+\delta_{n}^{2}+2 \delta_{n}<\varepsilon^{2} / 2$ for all $n \geq 1$, and such that the conclusion of the lemma is false for each $\delta_{n}$. Thus for each $n \geq 1$, there is a unital Banach $B_{n}$ and there exists elements $x_{n}, y_{n} \in B_{n}$ which satisfy $\left\|x_{n}\right\| \leq M,\left\|x_{n}^{2}-x_{n}\right\| \leq \delta_{n},\left\|x_{n}-y_{n}\right\| \leq \delta_{n}$ and such that for each $n \geq 1$,

(6) $\max \left\{\left\|\chi\left(y_{n}\right)-x_{n}\right\|,\left\|\chi\left(x_{n}\right)-x_{n}\right\|,\left\|\chi\left(y_{n}\right)-y_{n}\right\|,\left\|\chi\left(x_{n}\right)-\chi\left(y_{n}\right)\right\|\right\} \geq \varepsilon$.

Consider the unital Banach algebra $A:=\prod_{n} B_{n}$ consisting of all bounded sequences $b=\left(b_{n}\right)$ with $b_{n} \in B_{n}$ and norm $\|b\|=\sup _{n}\left\|b_{n}\right\|$. Let $J$ be the two-sided closed ideal of $A$ consisting of sequences $b=\left(b_{n}\right)$ such that $\lim _{n}\left\|b_{n}\right\|=0$ and let $q: A \rightarrow A / J$ denote the canonical quotient morphism. Then $A / J$ is a unital Banach algebra. Let us consider the elements $x=\left(x_{n}\right)$ and $y=\left(y_{n}\right)$ of $A$. By arguing as above we see that $S p(x) \cup S p(y) \subset V(\varepsilon)$ 
and that $x^{2}-x, y^{2}-y \in J$. It is then clear that $q(x)^{2}=q(x)=q(y)=q(y)^{2}$ and by the functoriality of the Riesz calculus, $q(x)=\chi(q(x))=q(\chi(x))$ and $q(y)=\chi(q(y))=q(\chi(y))$. This shows that $\{x-\chi(x), y-\chi(y), x-$ $\chi(y), \chi(x)-\chi(y)\} \subset J$. It follows that $\lim _{n}\left\|x_{n}-\chi\left(x_{n}\right)\right\|=\lim _{n} \| y_{n}-$ $\chi\left(y_{n}\right)\left\|=\lim _{n}\right\| x_{n}-\chi\left(y_{n}\right)\left\|=\lim _{n}\right\| \chi\left(x_{n}\right)-\chi\left(y_{n}\right) \|=0$. This contradicts (6).

Lemma 2.4. For any $M \geq 1$ and $0<\varepsilon<1 / 2$ there is $\theta=\theta(M, \varepsilon)>0$ with the following property. Let $B$ be a unital Banach algebra and let $E$ be a Banach right $B$-module. Let $e, f \in L_{B}(E)$ and $h \in K_{B}(E)$ be idempotents such that

(i) $e-f \in K_{B}(E)$,

(ii) $\|e\| \leq M,\|f\| \leq M,\|h\| \leq M$,

(iii) $\|[e, h]\| \leq \theta,\|[f, h]\| \leq \theta,\|(1-h)(e-f)(1-h)\| \leq \theta$.

Then

(a) $S p(h e h) \cup S p(h f h) \subset V(\varepsilon)$,

(b) $\|h e h-\chi(h e h)\|<\varepsilon, \quad\|h f h-\chi(h f h)\|<\varepsilon$

(c) $[e, f]=[\chi(h e h), \chi(h f h)] \in K K^{b a n}(\mathbb{C}, B) \cong K_{0}(B)$.

Proof. We may assume that $\varepsilon<1 /\left(8(M+1)^{3}+4\right)$. Let $\delta$ be the two-variable function given by Lemma 2.3. We show that if

$$
0<\theta<\min \left\{\varepsilon, \frac{\delta\left(M^{3}, \varepsilon\right)}{M^{3}}, \frac{\delta\left((M+1)^{3}, \varepsilon\right)}{(M+1)^{3}}, \frac{\delta\left(2(M+1)^{3}, \varepsilon\right)}{2 M}\right\}
$$

then $\theta$ satisfies the statement of the Lemma 2.4. Set

$$
x=h e h, x^{\prime}=(1-h) e(1-h), y=h f h, y^{\prime}=(1-h) f(1-h) .
$$

We have a series of straightforward estimates:

$$
\begin{gathered}
\|x\| \leq M^{3}, \quad\left\|x^{\prime}\right\| \leq(M+1)^{2} M, \quad\|y\| \leq M^{3}, \quad\left\|y^{\prime}\right\| \leq(M+1)^{2} M \\
\left\|x+x^{\prime}\right\| \leq 2(M+1)^{3}, \quad\left\|y+y^{\prime}\right\| \leq 2(M+1)^{3} \\
\left\|e-\left(x+x^{\prime}\right)\right\|=\|h(h e-e h)+(e h-h e) h\| \leq 2 M \theta<\delta\left(2(M+1)^{3}, \varepsilon\right) \\
\left\|f-\left(y+y^{\prime}\right)\right\|<\delta\left(2(M+1)^{3}, \varepsilon\right) \\
\left\|x^{\prime}-y^{\prime}\right\|=\|(1-h)(e-f)(1-h)\| \leq \theta<\varepsilon \\
\left\|x^{2}-x\right\|=\|h e(h e-e h) h\| \leq M^{3} \theta<\delta\left(M^{3}, \varepsilon\right) \\
\left\|x^{\prime 2}-x^{\prime}\right\|=\|\left((1-h) e(h e-e h)(1-h) \| \leq(M+1)^{3} \theta<\delta\left((M+1)^{3}, \varepsilon\right)\right. \\
\left\|y^{2}-y\right\|<\delta\left(M^{3}, \varepsilon\right), \quad\left\|y^{\prime 2}-y^{\prime}\right\|<\delta\left((M+1)^{3}, \varepsilon\right) .
\end{gathered}
$$


These estimates enable us to apply Lemma 2.3 and obtain that both $S p(x)$ an $S p(y)$ are contained in $V(\varepsilon)$ and moreover

$$
\begin{gathered}
\|x-\chi(x)\|<\varepsilon, \quad\left\|x^{\prime}-\chi\left(x^{\prime}\right)\right\|<\varepsilon, \quad\|y-\chi(y)\|<\varepsilon, \quad\left\|y^{\prime}-\chi\left(y^{\prime}\right)\right\|<\varepsilon \\
\left\|\chi\left(x^{\prime}\right)-\chi\left(y^{\prime}\right)\right\|<\varepsilon, \quad\left\|e-\chi\left(x+x^{\prime}\right)\right\|<\varepsilon, \quad\left\|f-\chi\left(y+y^{\prime}\right)\right\|<\varepsilon .
\end{gathered}
$$

Note that $\chi(x), \chi(y) \in K_{B}(E), x^{\prime}-y^{\prime} \in K_{B}(E)$ and hence $\chi\left(x^{\prime}\right)-\chi\left(y^{\prime}\right) \in$ $K_{B}(E)$. Recall that $x \in h B h$ and $x^{\prime} \in(1-h) B(1-h)$ and hence $\chi(x+$ $\left.x^{\prime}\right)=\chi(x)+\chi\left(x^{\prime}\right)$ and $\chi(x) \chi\left(x^{\prime}\right)=\chi\left(x^{\prime}\right) \chi(x)=0$. Similar properties hold for $y$ and $y^{\prime}$ and we will use these in the sequel. Since $\|e\| \leq M$, $\left\|\chi\left(x+x^{\prime}\right)\right\| \leq\|e\|+\varepsilon<M+1,\|f\| \leq M,\left\|\chi\left(y+y^{\prime}\right)\right\| \leq\|f\|+\varepsilon<M+1$, and $\varepsilon<1 /(8(M+1)+4)$, we deduce from Lemma 2.2 that

$$
[e, f]=\left[\chi\left(x+x^{\prime}\right), \chi\left(y+y^{\prime}\right)\right]=\left[\chi(x)+\chi\left(x^{\prime}\right), \chi(y)+\chi\left(y^{\prime}\right)\right] .
$$

On the other hand,

$$
\left[\chi(x)+\chi\left(x^{\prime}\right), \chi(y)+\chi\left(y^{\prime}\right)\right]=[\chi(x), \chi(y)]+\left[\chi\left(x^{\prime}\right), \chi\left(y^{\prime}\right)\right]=[\chi(x), \chi(y)],
$$

since $\left[\chi\left(x^{\prime}\right), \chi\left(y^{\prime}\right)\right]=\left[\chi\left(x^{\prime}\right), \chi\left(x^{\prime}\right)\right]=0$, also by Lemma 2.2 which we can apply since we have $\left\|\chi\left(x^{\prime}\right)\right\| \leq\left\|x^{\prime}\right\|+\varepsilon<(M+1)^{3},\left\|\chi\left(y^{\prime}\right)\right\| \leq\left\|y^{\prime}\right\|+\varepsilon<$ $(M+1)^{3}$ and

$$
\left\|\chi\left(x^{\prime}\right)-\chi\left(y^{\prime}\right)\right\|<\varepsilon<1 /\left(8(M+1)^{3}+4\right) .
$$

We conclude that $[e, f]=[\chi(x), \chi(y)]$.

The following description of a special case of the cap product (3) plays an important role in the paper.

Proposition 2.5. Let $A, B$ be unital Banach algebras. Suppose that an element $\alpha \in K K^{\text {ban }}(A, \mathbb{C})$ is given by the class of a triplet

$$
\left(H \oplus H, \varphi=\left(\begin{array}{cc}
\varphi_{0} & 0 \\
0 & \varphi_{1}
\end{array}\right),\left(\begin{array}{ll}
0 & 1 \\
1 & 0
\end{array}\right)\right),
$$

where $H$ is a Banach space. Let $p \in A \otimes B$ be an idempotent. Let $\widetilde{\varphi}_{i}:=$ $\varphi_{i} \otimes i d_{B}: A \otimes B \rightarrow L(H) \otimes B$. Let $\widetilde{e} \in M_{r}(\mathbb{C}) \otimes B \subset L(H) \otimes B$ be an idempotent $\left(M_{r}(\mathbb{C}) \cong L\left(\mathbb{C}^{m}\right) \subset L(H)\right.$ is induced by a choice of embedding $\left.\mathbb{C}^{m} \subset H\right)$. Suppose that there is $M>0$ and $0<\varepsilon<1 / 2$ such that

(i) $\|\widetilde{e}\| \leq M, \quad\left\|\widetilde{\varphi}_{i}(p)\right\| \leq M, \quad i=0,1$,

(ii) $\left\|\left[\widetilde{\varphi}_{i}(p), \widetilde{e}\right]\right\|<\theta(M, \varepsilon), \quad i=0,1$,

(iii) $\left\|(1-\widetilde{e})\left(\widetilde{\varphi}_{0}(p)-\widetilde{\varphi}_{1}(p)\right)(1-\widetilde{e})\right\|<\theta(M, \varepsilon)$, 
where $\theta(M, \varepsilon)$ is given by Lemma 2.4.

Then the idempotents $q_{i}:=\chi\left(\widetilde{e} \widetilde{\varphi}_{i}(p) \widetilde{e}\right) \in M_{r}(B)$ are well defined, they satisfy

$$
\left\|q_{i}-\widetilde{e} \widetilde{\varphi}_{i}(p) \widetilde{e}\right\|<\varepsilon, \quad i=0,1
$$

and the cap product

$$
K K^{b a n}(\mathbb{C}, A \otimes B) \times K K^{b a n}(A, \mathbb{C}) \rightarrow K K^{b a n}(\mathbb{C}, B)
$$

satisfies

$$
[p] \circ\left(\alpha \otimes 1_{B}\right)=\left[q_{0}\right]-\left[q_{1}\right],
$$

under the isomorphism $K K^{\text {ban }}(\mathbb{C}, B) \cong K_{0}(B)$. More generally, an entirely similar statement holds for idempotents $p \in M_{m}(\mathbb{C}) \otimes A \otimes B$.

Proof. Let $j$ be the linear contractive map $j: L(H) \otimes B \rightarrow L_{B}(H \otimes B)$ and let $\widetilde{\varphi}_{i}=j \circ \widetilde{\varphi}_{i}, i=0,1$. From the definition of the product (2) as described in $(5)$, it follows that $[p] \circ\left(\alpha \otimes 1_{B}\right)=\left[\widetilde{\widetilde{\varphi}}_{0}(p), \widetilde{\widetilde{\varphi}}_{1}(p)\right]$. On the other hand, since $j$ is contractive, we deduce from the hypotheses of the proposition that

$$
\begin{gathered}
\|\widetilde{\widetilde{e}}\| \leq M, \quad\left\|\widetilde{\widetilde{\varphi}}_{i}(p)\right\| \leq M, \quad i=0,1 \\
\left\|\left[\widetilde{\widetilde{\varphi}}_{i}(p), \widetilde{\widetilde{e}}\right]\right\|<\theta(M, \varepsilon), \quad i=0,1 \\
\left\|(1-\widetilde{\widetilde{e}})\left(\widetilde{\widetilde{\varphi}}_{0}(p)-\widetilde{\widetilde{\varphi}}_{1}(p)\right)(1-\widetilde{\widetilde{e}})\right\|<\theta(M, \varepsilon) .
\end{gathered}
$$

These estimates enable us to apply Lemma 2.4 and obtain that

$$
\begin{aligned}
{\left[\widetilde{\widetilde{\varphi}}_{0}(p), \widetilde{\widetilde{\varphi}}_{1}(p)\right] } & =\left[\chi\left(\widetilde{\widetilde{e}} \widetilde{\varphi}_{0}(p) \widetilde{\widetilde{e}}\right), \chi\left(\widetilde{\widetilde{e}} \widetilde{\varphi}_{1}(p) \widetilde{\widetilde{e}}\right)\right] \\
& =\left[j\left(\chi\left(\widetilde{e} \widetilde{\varphi}_{0}(p) \widetilde{e}\right)\right), j\left(\chi\left(\widetilde{e} \widetilde{\varphi}_{1}(p) \widetilde{e}\right)\right)\right]=\left[j\left(q_{0}\right), j\left(q_{1}\right)\right]
\end{aligned}
$$

in $K K^{\text {ban }}(\mathbb{C}, B)$. By [16, Thm. 1.2.8], the image of $\left[j\left(q_{0}\right), j\left(q_{1}\right)\right]$ in $K_{0}(B)$ is equal to $\left[q_{0}\right]-\left[q_{1}\right]$.

\section{Group Quasi-Representations And indeX theOrems}

Let $G$ be a discrete countable group. Consider the action of $G$ on $\ell^{1}(G)$ by multiplication, by viewing $G$ as a set of units of $\ell^{1}(G)$. Let $\widetilde{Y} \rightarrow Y$ be a principal $G$-bundle over a compact metrizable space $Y$. Let $\ell_{Y}$ be the "line bundle" $\widetilde{Y} \times{ }_{G} \ell^{1}(G) \rightarrow Y$ induced by the diagonal action of $G$. One can cover $Y$ by a finite family of open sets $\left(U_{\alpha}\right)_{\alpha \in I}$ in such a way that the bundle $\ell_{Y}$ is obtained by gluing the trivial bundles $U_{\alpha} \times \ell^{1}(G)$ via a constant cocycle $s_{\alpha \beta} \in G \subset \ell^{1}(G)$. Thus $s_{\alpha \beta} s_{\beta \gamma}=s_{\alpha \gamma}$ whenever $U_{\alpha} \cap U_{\beta} \cap U_{\gamma} \neq \emptyset$. If $\left(\chi_{\alpha}\right)_{\alpha \in I}$ are positive continuous functions with $\chi_{\alpha}$ supported in $U_{\alpha}$ and such that $\sum_{\alpha \in I} \chi_{\alpha}^{2}=1$, then the finitely generated 
projective right Banach $C(Y) \otimes \ell^{1}(G)$-module of continuous sections in $\ell_{Y}$ is represented by an idempotent

$$
P=\sum_{\alpha, \beta \in I} e_{\alpha \beta} \otimes \chi_{\alpha} \chi_{\beta} \otimes s_{\alpha \beta} \in M_{m} \otimes C(Y) \otimes \ell^{1}(G) .
$$

Here $m=|I|$ and $\left(e_{\alpha \beta}\right)$ is the canonical matrix unit of $M_{m}(\mathbb{C})$.

Definition 3.1. Let $G$ be a discrete countable group and let $B$ be a unital Banach algebra. Let $\mathcal{G} \subset G$ be a finite subset and let $\omega>0$. A map $\pi: G \rightarrow$ $B$ is called a $(\mathcal{G}, \omega)$-representation if it satisfies the following conditions: (i) $\pi(1)=1$ and $\|\pi(s)\| \leq 1$ for all $s \in G$; (ii) $\|\pi(s t)-\pi(s) \pi(t)\|<\omega$ for all $s, t \in \mathcal{G}$.

If $B$ is a unital $C^{*}$-algebra, a map $\pi: G \rightarrow B$ is called a $(\mathcal{G}, \omega)$-unitary representation if it satisfies (i), (ii) from above and (iii) $\left\|\pi\left(s^{-1}\right)-\pi(s)^{*}\right\|<\omega$ for all $s \in \mathcal{G}$. Note that if $\omega<1$ and $\left\{s, s^{-1}\right\} \subset \mathcal{G}$, then $\pi(s) \in G L(B)$.

One can extend $\pi$ to a linear contraction $\pi: \ell^{1}(G) \rightarrow B$ by $\pi\left(\sum_{s \in G} \lambda_{s} s\right)=$ $\sum_{s \in G} \lambda_{s} \pi(s)$.

Let $B$ be a unital Banach algebra. An element $z \in K K(C(Y), \mathbb{C})$ gives rise to an element $z \otimes 1_{B} \in K K^{\text {ban }}(C(Y) \otimes B, B)$ which induces via the cap product (3) a map $K_{0}(C(Y) \otimes B) \rightarrow K_{0}(B), x \mapsto x \circ\left(z \otimes 1_{B}\right)$. We work with the following $\ell^{1}$-version of the assembly map:

$$
\mu: K K(C(Y), \mathbb{C}) \rightarrow K_{0}\left(\ell^{1}(G)\right), \quad \mu(z)=[P] \circ\left(z \otimes 1_{\ell^{1}(G)}\right) .
$$

If $\pi: \ell^{1}(G) \rightarrow B$ is a unital contractive morphism of Banach algebras, then there is a commutative diagram

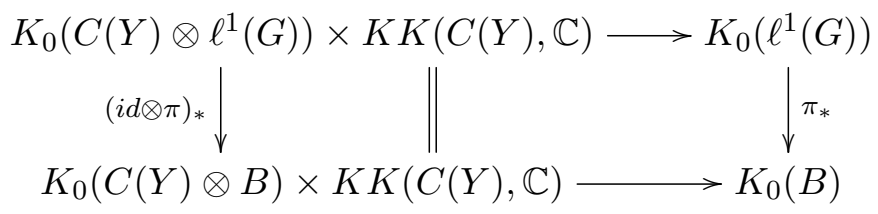

The following result extends this property to quasi-representations (starting from scratch), see Definitions 2.1 and 3.1.

Theorem 3.2. Let $z \in K K(C(Y), \mathbb{C})$ and let $q_{0}, q_{1}$ be idempotents in matrices over $\ell^{1}(G)$ such that $\mu(z)=[P] \circ\left(z \otimes 1_{\ell^{1}(G)}\right)=\left[q_{0}\right]-\left[q_{1}\right]$. Then there exist a finite subset $\mathcal{G} \subset G$ and $\omega>0$ (which depend on the representatives $P, q_{0}$ and $\left.q_{1}\right)$, such that if $B$ is a unital Banach algebra and $\pi: G \rightarrow B$ is any $(\mathcal{G}, \omega)$-representation, then

$$
\pi_{\sharp}\left(q_{0}\right)-\pi_{\sharp}\left(q_{1}\right)=\left(\operatorname{id}_{C(Y)} \otimes \pi\right)_{\sharp}(P) \circ\left(z \otimes 1_{B}\right) .
$$


With the convention that follows Definition 2.1, we could have written $\pi_{\sharp}(\mu(z))$ in place of $\pi_{\sharp}\left(q_{0}\right)-\pi_{\sharp}\left(q_{1}\right)$.

Proof. Given two pairs of idempotents $q_{0}, q_{1}$ and $q_{0}^{\prime}, q_{1}^{\prime}$ in matrices over $\ell^{1}(G)$ such that $\left[q_{0}\right]-\left[q_{1}\right]=\left[q_{0}^{\prime}\right]-\left[q_{1}^{\prime}\right]$, there exist $\mathcal{G}_{0}$ and $\omega_{0}>0$ such that if $\mathcal{G}_{0} \subset \mathcal{G}$ and $\omega<\omega_{0}$, then for any $(\mathcal{G}, \omega)$-representation $\pi: G \rightarrow B$, one has $\pi_{\sharp}\left(q_{0}\right)-\pi_{\sharp}\left(q_{1}\right)=\pi_{\sharp}\left(q_{0}^{\prime}\right)-\pi_{\sharp}\left(q_{1}^{\prime}\right)$. Thus it suffices to prove the theorem for a pair of idempotents $q_{0}, q_{1}$ of our choice with $\mu(z)=\left[q_{0}\right]-\left[q_{1}\right]$.

Before we can exhibit $\mathcal{G}$ and $\omega$ we need to choose carefully three constants $\varepsilon_{1}, \varepsilon_{2}$ and $\eta$. Let us regard the numbers $\delta(M, \varepsilon)$ and $\theta(M, \varepsilon)$ given by the Lemma 2.3 and respectively Lemma 2.4 as functions of the variables $M$ and $\varepsilon$. For $m>0$ as in the definition of $P$ and $0<\varepsilon<1 / 3$ we choose $0<\varepsilon_{1}<1 / 2$ such that

$$
\varepsilon_{1}<\delta\left(m^{2}, \varepsilon\right) .
$$

Next we choose $\varepsilon_{2}>0$ such that

$$
\varepsilon_{2}<\min \left\{\varepsilon, \frac{1}{4} \theta\left(m^{2}+1, \varepsilon\right)\right\} .
$$

Then we fix a number $0<\eta<1$ such that

$$
\eta<\min \left\{\frac{1}{2 m^{3}} \delta\left(m^{2}, \varepsilon\right), \frac{1}{m^{2}} \theta\left(m^{2}, \varepsilon_{1}\right), \frac{1}{2 m^{2}} \theta\left(m^{2}+1, \varepsilon\right)\right\} .
$$

We are going to describe the construction of the idempotents $q_{0}$ and $q_{1}$ that are convenient to work with. By the Cuntz-picture of the KK-groups [3], any element $z \in K K(C(Y), \mathbb{C})$ is represented by a pair of $*$-representations $\varphi_{0}, \varphi_{1}: C(Y) \rightarrow L(H)$ such that $\varphi_{0}(a)-\varphi_{1}(a) \in K(H)$ for all $a \in C(Y)$. Correspondingly, we have Banach algebra morphisms

$$
\widetilde{\varphi}_{i}=\mathrm{id}_{m} \otimes \varphi_{i} \otimes \mathrm{id}_{\ell^{1}(G)}: M_{m} \otimes C(Y) \otimes \ell^{1}(G) \longrightarrow M_{m} \otimes L(H) \otimes \ell^{1}(G) .
$$

By Voiculescu's theorem, $\varphi_{1}$ is a compact perturbation of a $*$-representation of $C(Y)$ which is a direct sum of one-dimensional representations. Therefore, there is an approximate unit $\left(e_{n}\right)_{n}$ of $K(H)$ consisting of projections such that $\lim _{n}\left\|\left[\varphi_{1}(a), e_{n}\right]\right\|=0$ for all $a \in C(Y)$. Since $\varphi_{0}(a)-\varphi_{1}(a) \in K(H)$ for all $a \in C(Y)$, it follows that there is a projection $e \in M_{r}(\mathbb{C})=L\left(\mathbb{C}^{r}\right) \subset$ $L(H)$ such that

$$
\left\|\left[\varphi_{i}(a), e\right]\right\|<\eta, \quad\left\|(1-e)\left(\varphi_{0}(a)-\varphi_{1}(a)\right)(1-e)\right\|<\eta,
$$

for all $a \in\left\{\chi_{\alpha} \chi_{\beta} ; \alpha, \beta \in I\right\}$ and $i=0,1$.

Let $\widetilde{e}:=1_{m} \otimes e \otimes 1_{\ell^{1}(G)}$. Then $\|\widetilde{e}\| \leq 1$. Let us note that

$$
\left\|\widetilde{\varphi}_{i}(P)\right\|=\left\|\sum_{\alpha, \beta \in I} e_{\alpha \beta} \otimes \varphi_{i}\left(\chi_{\alpha} \chi_{\beta}\right) \otimes s_{\alpha \beta}\right\| \leq m^{2},
$$


since $\left\|s_{\alpha \beta}\right\| \leq 1$. Next we verify that

(15) $\left\|\left[\widetilde{\varphi}_{i}(P), \widetilde{e}\right]\right\|<m^{2} \eta$, and $\left\|(1-\widetilde{e})\left(\widetilde{\varphi}_{0}(P)-\widetilde{\varphi}_{1}(P)\right)(1-\widetilde{e})\right\|<m^{2} \eta$.

Using (14) we have

$$
\left\|\left[\widetilde{\varphi}_{i}(P), \widetilde{e}\right]\right\|=\left\|\sum_{\alpha, \beta \in I} e_{\alpha \beta} \otimes\left[\varphi_{i}\left(\chi_{\alpha} \chi_{\beta}\right), e\right] \otimes s_{\alpha \beta}\right\|<m^{2} \eta .
$$

For the other estimate, we write $\left\|(1-\widetilde{e})\left(\widetilde{\varphi}_{0}(P)-\widetilde{\varphi}_{1}(P)\right)(1-\widetilde{e})\right\|$ as

$$
\left\|\sum_{\alpha, \beta \in I} e_{\alpha \beta} \otimes(1-e)\left(\varphi_{0}\left(\chi_{\alpha} \chi_{\beta}\right)-\varphi_{1}\left(\chi_{\alpha} \chi_{\beta}\right)\right)(1-e) \otimes s_{\alpha \beta}\right\|<m^{2} \eta .
$$

Since $m^{2} \eta<\theta\left(m^{2}, \varepsilon_{1}\right)$ by (13), it follows by Proposition 2.5 that if we set $x_{i}:=\widetilde{e} \widetilde{\varphi}_{i}(P) \widetilde{e}$, then $S p\left(x_{i}\right) \subset V(1 / 2)$ and $q_{i}:=\chi\left(x_{i}\right) \in M_{m} \otimes M_{r} \otimes \ell^{1}(G)$ are idempotents such that

$$
\begin{gathered}
\left\|x_{i}-q_{i}\right\|<\varepsilon_{1}, \quad i=0,1, \\
\mu(z)=[P] \circ\left(z \otimes 1_{\ell^{1}(G)}\right)=\left[q_{0}\right]-\left[q_{1}\right] \in K_{0}\left(\ell^{1}(G)\right) .
\end{gathered}
$$

We now fix these idempotents $q_{i}=\chi\left(x_{i}\right), i=0,1$ and we will prove that if $\mathcal{G}=\left\{s_{\alpha \beta}: \alpha, \beta \in I\right\}$ and $\omega>0$ is such that

$$
\omega<\min \left\{\frac{1}{2 m^{3}} \delta\left(m^{2}, \varepsilon\right), \frac{1}{m^{3}} \delta\left(m^{2}, \varepsilon_{2}\right)\right\}
$$

then $\mathcal{G}$ and $\omega$ satisfy the statement of the theorem.

First we want to compute $\pi_{\sharp}\left(q_{0}\right)-\pi_{\sharp}\left(q_{1}\right)$ where $\pi: \ell^{1}(G) \rightarrow B$ is the canonical extension of a unital map $\pi: G \rightarrow B$ which satisfies $\|\pi(s)\| \leq 1$ for all $s \in G$ and

$$
\|\pi(s t)-\pi(s) \pi(t)\|<\omega \text { for all } s, t \in \mathcal{G} .
$$

Let $\widetilde{\pi}:=\mathrm{id}_{m} \otimes \mathrm{id}_{r} \otimes \pi: M_{m} \otimes M_{r} \otimes \ell^{1}(G) \rightarrow M_{m} \otimes M_{r} \otimes B$ and recall that

$$
x_{i}=\widetilde{e} \widetilde{\varphi}_{i}(P) \widetilde{e}=\sum_{\alpha, \beta \in I} e_{\alpha \beta} \otimes e \varphi_{i}\left(\chi_{\alpha} \chi_{\beta}\right) e \otimes s_{\alpha \beta}
$$

Since $\tilde{\pi}$ is contractive, $\left\|\widetilde{\pi}\left(x_{i}\right)\right\| \leq m^{2}$. Moreover, it follows from (16) and (11) that

$$
\left\|\tilde{\pi}\left(q_{i}\right)-\tilde{\pi}\left(x_{i}\right)\right\|<\varepsilon_{1}<\delta\left(m^{2}, \varepsilon\right) .
$$

We are going to verify that

$$
\left\|\widetilde{\pi}\left(x_{i}\right)^{2}-\widetilde{\pi}\left(x_{i}\right)\right\|<\delta\left(m^{2}, \varepsilon\right), \quad i=0,1 .
$$

To this purpose let us consider the following expressions:

$$
U_{i}=\sum_{\alpha, \beta, \gamma} e_{\alpha \gamma} \otimes e \varphi_{i}\left(\chi_{\alpha} \chi_{\beta}\right) e \varphi_{i}\left(\chi_{\beta} \chi_{\gamma}\right) e \otimes \pi\left(s_{\alpha \beta}\right) \pi\left(s_{\beta \gamma}\right),
$$




$$
\begin{gathered}
V_{i}=\sum_{\alpha, \beta, \gamma} e_{\alpha \gamma} \otimes e \varphi_{i}\left(\chi_{\alpha} \chi_{\beta}\right) \varphi_{i}\left(\chi_{\beta} \chi_{\gamma}\right) e \otimes \pi\left(s_{\alpha \beta}\right) \pi\left(s_{\beta \gamma}\right), \\
W_{i}=\tilde{\pi}\left(x_{i}\right)=\sum_{\alpha, \beta, \gamma} e_{\alpha \gamma} \otimes e \varphi_{i}\left(\chi_{\alpha} \chi_{\beta}^{2} \chi_{\gamma}\right) e \otimes \pi\left(s_{\alpha \gamma}\right),
\end{gathered}
$$

and observe that $\tilde{\pi}\left(x_{i}\right)^{2}-\tilde{\pi}\left(x_{i}\right)=\left(U_{i}-V_{i}\right)+\left(V_{i}-W_{i}\right)$, that $\left\|U_{i}-V_{i}\right\|<m^{3} \eta$ by (14) and that $\left\|V_{i}-W_{i}\right\|<m^{3} \omega$ by (19). It follows from (13) and (18) that

$$
\left\|\widetilde{\pi}\left(x_{i}\right)^{2}-\tilde{\pi}\left(x_{i}\right)\right\| \leq m^{3} \eta+m^{3} \omega<\delta\left(m^{2}, \varepsilon\right) .
$$

Thus we have verified the inequality $(22)$.

Due to the estimates (21), (22) and $\left\|\widetilde{\pi}\left(x_{i}\right)\right\| \leq m^{2}$, we can apply Lemma 2.3 to deduce that for any map $\pi: G \rightarrow B$ as in the statement, we have

$$
\begin{gathered}
\left\|\chi\left(\widetilde{\pi}\left(q_{i}\right)\right)-\tilde{\pi}\left(x_{i}\right)\right\|<\varepsilon, \\
\pi_{\sharp}\left(q_{0}\right)-\pi_{\sharp}\left(q_{1}\right)=\left[\chi\left(\widetilde{\pi}\left(q_{0}\right)\right)\right]-\left[\chi\left(\widetilde{\pi}\left(q_{1}\right)\right)\right] .
\end{gathered}
$$

In the second part of the proof we are going to compute $\left(\operatorname{id}_{C(Y)} \otimes \pi\right)_{\sharp}(P) \circ$ $\left(z \otimes 1_{B}\right)$ by using again Lemma 2.3 and Proposition 2.5. The result of this calculation is given in (31). The proof will be completed by comparing the expressions from (24) and (31). If we set

$x:=\left(\operatorname{id}_{m} \otimes \operatorname{id}_{C(Y)} \otimes \pi\right)(P)=\sum_{\alpha, \beta \in I} e_{\alpha \beta} \otimes \chi_{\alpha} \chi_{\beta} \otimes \pi\left(s_{\alpha \beta}\right) \in M_{m} \otimes C(Y) \otimes B$,

then $\|x\| \leq m^{2}$. Since

$$
x^{2}-x=\sum_{\alpha, \delta} e_{\alpha \delta} \otimes \sum_{\beta} \chi_{\alpha} \chi_{\beta}^{2} \chi_{\delta} \otimes\left(\pi\left(s_{\alpha \beta}\right) \pi\left(s_{\beta, \delta}\right)-\pi\left(s_{\alpha \delta}\right)\right),
$$

we obtain from (18) and (19) the estimate

$$
\left\|x^{2}-x\right\| \leq m^{3} \omega<\delta\left(m^{2}, \varepsilon_{2}\right) .
$$

By definition, $\left(\operatorname{id}_{C(Y)} \otimes \pi\right)_{\sharp}(P)=[h]$, where $h$ is the idempotent $h=\chi(x)$ given by Lemma 2.3. Thus $\left(\operatorname{id}_{C(Y)} \otimes \pi\right)_{\sharp}(P) \circ\left(z \otimes 1_{B}\right)=[h] \circ\left(z \otimes 1_{B}\right)$, where

$$
\|h-x\|=\|\chi(x)-x\|<\varepsilon_{2} .
$$

In order to compute $[h] \circ\left(z \otimes 1_{B}\right)$, we need to consider the maps

$$
\Phi_{i}=\mathrm{id}_{m} \otimes \varphi_{i} \otimes \mathrm{id}_{B}: M_{m} \otimes C(Y) \otimes B \rightarrow M_{m} \otimes L(H) \otimes B .
$$

Since $\varphi_{i}$ is contractive, so is $\Phi_{i}$ and hence by (26) we obtain that

$$
\left\|\mid \Phi_{i}(h)-\Phi_{i}(x)\right\|<\varepsilon_{2}, \quad i=0,1,
$$

and hence $\left\|\Phi_{i}(h)\right\| \leq\left\|\Phi_{i}(x)\right\|+\varepsilon_{2}<m^{2}+1, i=0,1$. 
We are going to show that the idempotents $\Phi_{i}(h)$ and $E:=1_{m} \otimes e \otimes 1_{B}$ satisfy the assumptions of Proposition 2.5 with $\theta=\theta\left(m^{2}+1, \varepsilon\right)$. Thus we must verify that

$\left\|\left[\Phi_{i}(h), E\right]\right\|<\theta\left(m^{2}+1, \varepsilon\right) \quad$ and $\quad\left\|(1-E)\left(\Phi_{0}(h)-\Phi_{1}(h)\right)(1-E)\right\|<\theta\left(m^{2}, \varepsilon\right)$.

We first estimate $\left\|\left[\Phi_{i}(x), E\right]\right\|$ and $\left\|(1-E)\left(\Phi_{0}(x)-\Phi_{1}(x)\right)(1-E)\right\|$. Since

$$
\Phi_{i}(x)=\sum_{\alpha, \beta \in I} e_{\alpha \beta} \otimes \varphi_{i}\left(\chi_{\alpha} \chi_{\beta}\right) \otimes \pi\left(s_{\alpha \beta}\right),
$$

using (14) we have

$$
\begin{gathered}
{\left[\Phi_{i}(x), E\right]=\left\|\sum_{\alpha, \beta \in I} e_{\alpha \beta} \otimes\left[e, \varphi_{i}\left(\chi_{\alpha} \chi_{\beta}\right)\right] \otimes \pi\left(s_{\alpha \beta}\right)\right\|<m^{2} \eta, \quad \text { and }} \\
\left\|(1-E)\left(\Phi_{0}(x)-\Phi_{1}(x)\right)(1-E)\right\|<m^{2} \eta
\end{gathered}
$$

as it becomes clear after writing it in the form

$$
\left\|\sum_{\alpha, \beta \in I} e_{\alpha \beta} \otimes(1-e)\left(\varphi_{0}\left(\chi_{\alpha} \chi_{\beta}\right)-\varphi_{1}\left(\chi_{\alpha} \chi_{\beta}\right)\right)(1-e) \otimes \pi\left(s_{\alpha \beta}\right)\right\| .
$$

From $(27),(29)$ and (30) we deduce that $\left\|(1-E)\left(\Phi_{0}(h)-\Phi_{1}(h)\right)(1-E)\right\|$ and $\left\|\left[\Phi_{i}(h), E\right]\right\|, i=0,1$ are dominated by $m^{2} \eta+2 \varepsilon_{2}$ and hence they are smaller than $\theta\left(m^{2}+1, \varepsilon\right)$ by (12) and (13). Applying Proposition 2.5 we obtain that

$$
[h] \circ\left(z \otimes 1_{B}\right)=\left[\chi\left(E \Phi_{0}(h) E\right)\right]-\left[\chi\left(E \Phi_{1}(h) E\right)\right],
$$

as well as the estimate

$$
\left\|\chi\left(E \Phi_{i}(h) E\right)-E \Phi_{i}(h) E\right\|<\varepsilon .
$$

On the other hand, from (26) and (32) we see that

$$
\left\|\chi\left(E \Phi_{i}(h) E\right)-E \Phi_{i}(x) E\right\|<\varepsilon+\varepsilon_{2}<2 \varepsilon .
$$

It is important to note that $E \Phi_{i}(x) E=\widetilde{\pi}\left(x_{i}\right)$, as it follows by comparing (20) and (28). Consequently, from (23) and (33) we deduce that

$$
\| \chi\left(\widetilde{\pi}\left(q_{i}\right)\right)-\chi\left(E \Phi_{i}(h) E\right) \mid<3 \varepsilon<1 .
$$

This shows that $\left[\chi\left(\widetilde{\pi}\left(q_{i}\right)\right)\right]=\left[\chi\left(E \Phi_{i}(h) E\right)\right]$ in $K_{0}(B), i=0,1$. Therefore from (24) and (31) we obtain that $[h] \circ\left(z \otimes 1_{B}\right)=\pi_{\sharp}\left(q_{0}\right)-\pi_{\sharp}\left(q_{1}\right)$.

Let $G$ be a discrete countable group and let $j: \ell^{1}(G) \rightarrow C^{*}(G)$ be the canonical $*$-homomorphism. 
Lemma 3.3. Let $x, y \in K_{0}\left(\ell^{1}(G)\right)$ be such that $j_{*}(x)=j_{*}(y)$ in $K_{0}\left(C^{*}(G)\right.$. Then there exist a finite subset $\mathcal{G}$ of $G$ and $\omega>0$ with the property that for any unital $C^{*}$-algebra $B$ and any $(\mathcal{G}, \omega)$-unitary representation, it follows that $\pi_{\sharp}(x)=\pi_{\sharp}(y)$.

Proof. The definitions of $\pi_{\sharp}(x), \pi_{\sharp}(y)$ depend on the choices of representatives for $x$ and $y$ as as explained in the comments following Definition 2.1. The set $\mathcal{G}$ and $\omega>0$ will also depend on these representatives.

For any given $(\mathcal{G}, \omega)$-unitary representation $\pi: G \rightarrow B$ we will denote also by $\pi$ its canonical linear extension $\pi: \ell^{1}(G) \rightarrow B$.

Fix idempotents $e_{i} \in M_{k}\left(\ell^{1}(G)\right), i=1,2,3,4$ such that $x=\left[e_{1}\right]-\left[e_{2}\right]$ and $y=\left[e_{3}\right]-\left[e_{4}\right]$. Seeking a contradiction, assume that the conclusion of the lemma is false. Then there exists a sequence of $\left(\mathcal{G}_{n}, \omega_{n}\right)$-unitary representations $\pi_{n}: G \rightarrow U\left(B_{n}\right)$ such that $\cup_{n} \mathcal{G}_{n}=G, 0<\omega_{n} \leq 1 / n$ with $\left\|x_{n}(i)^{2}-x_{n}(i)\right\|<1 / 4$, where $x_{n}(i)=\left(i d_{k} \otimes \pi_{n}\right)\left(e_{i}\right), i=1,2,3,4$, and such that $\pi_{n \sharp}(x) \neq \pi_{n \sharp}(y)$, or equivalently $\left[\chi\left(x_{n}(1)\right)\right]-\left[\chi\left(x_{n}(2)\right)\right] \neq\left[\chi\left(x_{n}(3)\right)\right]-$ $\left[\chi\left(x_{n}(4)\right)\right]$ in $K_{0}\left(B_{n}\right)$ for all $n \geq 1$. We can assemble the sequence $\left(\pi_{n}\right)$ to form a linear contractive map $\Phi: \ell^{1}(G) \rightarrow \prod_{n} B_{n}$. Let $q: \prod_{n} B_{n} \rightarrow B_{\infty}:=$ $\prod_{n} B_{n} / \sum_{n} B_{n}$ be the quotient map. Then $q \circ \Phi$ is a unital $*$-homomorphism. It follows that $q \circ \Phi$ factors through the universal group $\mathrm{C}^{*}$-algebra $C^{*}(G)$. In other words, there is a unital *-homomorphism $\Psi: C^{*}(G) \rightarrow B_{\infty}$ such that $q \circ \Phi=\Psi \circ j$. Since $j_{*}(x)=j_{*}(y)$ in $K_{0}\left(C^{*}(G)\right.$ by assumption, it follows that $(q \circ \Phi)_{*}(x)=(q \circ \Phi)_{*}(y)$ in $K_{0}\left(B_{\infty}\right)$. Let us note that by naturality of functional calculus, the idempotent $E_{i}=\left(\chi\left(x_{n}(i)\right)\right)_{n} \in M_{k}\left(\prod_{n} B_{n}\right)$ is a lifting of $\left(\operatorname{id}_{k} \otimes(q \circ \Phi)\right)\left(e_{i}\right)$. It follows that $q_{*}\left(\left[E_{1}\right]-\left[E_{2}\right]\right)=q_{*}\left(\left[E_{3}\right]-\left[E_{4}\right]\right)$ and hence $\left[E_{1}\right]-\left[E_{2}\right]$ and $\left[E_{3}\right]-\left[E_{4}\right]$ have the same image under the map

$$
K_{0}\left(\prod_{n} B_{n}\right) \rightarrow \prod_{n} K_{0}\left(B_{n}\right) / \sum_{n} K_{0}\left(B_{n}\right) .
$$

Therefore the sequences $\left(\left[\chi\left(x_{n}(1)\right)\right]-\left[\chi\left(x_{n}(2)\right)\right]\right)_{n}$ and $\left(\left[\chi\left(x_{n}(3)\right)\right]-\left[\chi\left(x_{n}(4)\right)\right]\right)_{n}$ could differ only by an element of $\sum_{n} K_{0}\left(B_{n}\right)$. This implies that $\pi_{n \sharp}(x)=$ $\pi_{n \sharp}(y)$ for all sufficiently large $n$, leading to the desired contradiction.

Definition 3.4. Let $G$ be a discrete countable group. Let $\widetilde{Y} \rightarrow Y$ be a principal $G$-bundle over a compact metrizable space $Y$. Following Lafforgue, we consider the map $\mu_{\ell^{1}}: K_{0}(Y) \rightarrow K_{0}\left(\ell^{1}(G)\right)$ which is defined as follows. Let $p \in \ell^{1}\left(G, C_{0}(\widetilde{Y})\right)$ be an idempotent constructed in the usual way from a cutoff function $c$, i.e. $p(s)(y)=\sqrt{c(y) c(s y)}, s \in G, y \in \tilde{Y}$. Let $j_{G}: K K_{G}^{b a n}\left(C_{0}(\widetilde{Y}), \mathbb{C}\right) \rightarrow K K^{\text {ban }}\left(\ell^{1}\left(G, C_{0}(\widetilde{Y})\right), \ell^{1}(G)\right)$ be the descent map of [16]. Let $z^{\prime} \in K K_{G}^{b a n}\left(C_{0}(\widetilde{Y}), \mathbb{C}\right)$ be the image of $z \in K_{0}(Y)=K K(C(Y), \mathbb{C})$ 
under the composition of the maps

$$
K K(C(Y), \mathbb{C}) \cong K K_{G}\left(C_{0}(\widetilde{Y}), \mathbb{C}\right) \rightarrow K K_{G}^{\text {ban }}\left(C_{0}(\tilde{Y}), \mathbb{C}\right) .
$$

Then by definition $\mu_{\ell^{1}}(z)=[p] \circ j_{G}\left(z^{\prime}\right)$.

We do not know whether or not $\mu$ coincide with $\mu_{\ell^{1}}$. We show that if $B$ is a $C^{*}$-algebra and if $\pi$ is a sufficiently multiplicative quasi-representation of $G$ in $B$, then one can replace $\mu$ by $\mu_{\ell^{1}}$ in Theorem 3.2.

Corollary 3.5. Let $z \in K_{0}(Y)$ and let $q_{0}, q_{1}$ be idempotents in matrices over $\ell^{1}(G)$ such that $\mu_{\ell^{1}}(z)=\left[q_{0}\right]-\left[q_{1}\right]$. Then there exist a finite subset $\mathcal{G} \subset G$ and $\omega>0$ such that if $B$ is a unital $C^{*}$-algebra and $\pi: G \rightarrow B$ is any $(\mathcal{G}, \omega)$-unitary representation, then

$$
\pi_{\sharp}\left(\mu_{\ell^{1}}(z)\right)=\pi_{\sharp}\left(q_{0}\right)-\pi_{\sharp}\left(q_{1}\right)=\left(\operatorname{id}_{C(Y)} \otimes \pi\right)_{\sharp}(P) \circ\left(z \otimes 1_{B}\right) .
$$

Proof. We use the fact that the natural map $K_{0}(Y) \rightarrow K_{0}\left(C^{*}(G)\right.$ ) (this is called the assembly map if $\widetilde{Y} \rightarrow Y$ is the universal covering of $Y$ ) has two equivalent descriptions $\mu^{\prime}$ and $\mu^{\prime \prime}$. The definition of $\mu^{\prime}$ is similar to (10):

$$
\mu^{\prime}: K K(C(Y), \mathbb{C}) \rightarrow K_{0}\left(C^{*}(G)\right), \quad \mu^{\prime}(z)=[P] \circ\left(z \otimes 1_{C^{*}(G)}\right) .
$$

The definition of $\mu^{\prime \prime}$ involves an idempotent $p \in C_{0}(\widetilde{Y}) \rtimes G$ (constructed from a cutoff function) and the image of $z \in K K(C(Y), \mathbb{C}) \cong K K_{G}\left(C_{0}(\widetilde{Y}), \mathbb{C}\right)$ under the descent map $j_{G}: K K_{G}\left(C_{0}(\widetilde{Y}), \mathbb{C}\right) \rightarrow K K\left(C_{0}(\widetilde{Y}) \rtimes G, C^{*}(G)\right)$.

$$
\mu^{\prime \prime}: K K(C(Y), \mathbb{C}) \rightarrow K_{0}\left(C^{*}(G)\right), \quad \mu^{\prime \prime}(z)=[p] \circ j_{G}(z) .
$$

It is known that $\mu^{\prime \prime}=\mu^{\prime}$, see [14], [26]. The definition of $\mu^{\prime \prime}$ is similar to that of $\mu_{\ell^{1}}$ and it is shown in [16] that if $j: \ell^{1}(G) \rightarrow C^{*}(G)$ is the canonical *-homomorphism, then $j_{*} \circ \mu_{\ell^{1}}=\mu^{\prime \prime}$. On the other hand by comparing equations (10) and (34) we see that $j_{*} \circ \mu=\mu^{\prime}$ and hence $j_{*} \circ \mu_{\ell^{1}}=j_{*} \circ \mu$. It follows then by Lemma 3.3 that $\pi_{\sharp}\left(\mu_{\ell^{1}}(z)\right)=\pi_{\sharp}(\mu(z))$. We conclude the proof by applying Theorem 3.2.

Let $M^{n}$ be a closed connected smooth manifold and let $G$ be a discrete countable group. Let $f: M \rightarrow B G$ be a continuous map to the classifying space of $G$ and let $\widetilde{M}$ be the pull-back of the universal cover $E G \rightarrow B G$. The diagonal action of $G$ on $\widetilde{M} \times \ell^{1}(G)$ defines a flat bundle $\widetilde{M} \times{ }_{G} \ell^{1}(G) \rightarrow M$, to which one can associate (as seen earlier (9)) an idempotent $P$ in matrices over $C(M) \otimes \mathbb{C}[G] \subset C(M) \otimes \ell^{1}(G)$. The construction of the analytic index $\operatorname{Ind}_{f, C^{*}(G)}(D)$ of an elliptic pseudodifferential operator $D$ on $M$ of Mishchenko and Kasparov has been refined by Connes and Moscovici and Lafforgue. The covering index of $D$ associated to the cover $\widetilde{M}$, denoted by $\operatorname{Ind}_{f, G}(D)$, was constructed in [6] as an element of 
$K_{0}(\mathbb{C}[G] \otimes \mathcal{R})$, where $\mathcal{R}$ is the algebra of rapid decay infinite matrices. We denote its image in $K_{0}\left(\ell^{1}(G)\right)$ by $\operatorname{Ind}_{f, \ell^{1}(G)}(D)$. On the other hand the map $\mu_{\ell^{1}}: K_{0}(M) \rightarrow K_{0}\left(\ell^{1}(G)\right)$ described in Definition 3.4 gives an element $\mu_{\ell^{1}}[D] \in K_{0}\left(\ell^{1}(G)\right)$. It is known that the images of $\operatorname{Ind}_{f, \ell^{1}(G)}(D)$ and $\mu_{\ell^{1}}[D]$ in $K_{0}\left(C^{*}(G)\right)$ coincide and they are both equal to $\operatorname{Ind}_{f, C^{*}(G)}(D)$ by [6], [4, Lemma 8, p.243] and [16]. If $h$ is the K-theory class of an idempotent in matrices over $C(M)$ we denote by $\operatorname{Ind}\left(D_{h}\right)=h \circ[D] \in \mathbb{Z}$ the index of $D$ twisted by $h$ given by the pairing $K^{0}(M) \times K_{0}(M) \rightarrow \mathbb{Z}$. The principal symbol of $D$ is denoted by $\sigma(D)$.

Let $B$ be a unital $C^{*}$-algebra which has a tracial state $\tau$. We shall use the Chern character $c h_{\tau}(E)$ defined for a smooth bundle $E$ on $M$ with fibers finitely generated projective modules over $B,[20],[24]$.

Let us fix idempotents $q_{0}, q_{1}$ in $M_{r}\left(\ell^{1}(G)\right)$ such that $\mu_{\ell^{1}}[D]=\left[q_{0}\right]-\left[q_{1}\right]$. Given a $(\mathcal{G}, \omega)$-unitary representation $\pi: G \rightarrow B$, where $B$ is a unital $\mathrm{C}^{*}$ algebra, we will write $\pi_{\sharp}\left(\mu_{\ell^{1}}[D]\right):=\pi_{\sharp}\left(q_{0}\right)-\pi_{\sharp}\left(q_{1}\right)$. Fix a smooth finitely generated projective $B$-module bundle $E_{\pi}$ over $M$ such that

$$
\left[E_{\pi}\right]=(1 \otimes \pi)_{\sharp}(P)
$$

in $K_{0}(C(M) \otimes B)$.

Theorem 3.6. Let $M^{n}$ be a closed connected smooth manifold and let $f$ : $M \rightarrow B G$ be a continuous map to the classifying space of a discrete countable group $G$. Let $D$ be an elliptic pseudodifferential operator on $M$. There exist a finite set $\mathcal{G} \subset G$ and $\omega>0$ (which depend on the choice of the representatives $q_{0}, q_{1}$ and $P$ ) such that if $B$ is a unital $C^{*}$-algebra endowed with a tracial state $\tau$ and $\pi: G \rightarrow B$ is a $(\mathcal{G}, \omega)$-unitary representation, then

$$
\tau\left(\pi_{\sharp}\left(\mu_{\ell^{1}}[D]\right)\right)=(-1)^{n}\left(\operatorname{ch}(\sigma(D)) \cup \operatorname{Td}(T M \otimes \mathbb{C}) \cup \operatorname{ch}_{\tau}\left(E_{\pi}\right)\left[T^{*} M\right] .\right.
$$

Proof. We apply Corollary 3.5 with $Y=M$ and $z=[D]$ to obtain that $\pi_{\sharp}\left(q_{0}\right)-\pi_{\sharp}\left(q_{1}\right)=(1 \otimes \pi)_{\sharp}(P) \circ\left([D] \otimes 1_{B}\right)=\left[E_{\pi}\right] \circ\left([D] \otimes 1_{B}\right)=: \operatorname{Ind}\left(D_{E_{\pi}}\right)$ and hence $\tau\left(\pi_{\sharp}\left(q_{0}\right)-\pi_{\sharp}\left(q_{1}\right)\right)=\tau\left(\operatorname{Ind}\left(D_{E_{\pi}}\right)\right)$. The latter expression is computed by the cohomological version of the Mishchenko-Fomenko index theorem, see [20], [24, Thm. 5.9]:

$$
\tau\left(\operatorname{Ind}\left(D_{E}\right)\right)=(-1)^{n}\left(\operatorname{ch}(\sigma(D)) \cup \operatorname{Td}(T M \otimes \mathbb{C}) \cup \operatorname{ch}_{\tau}(E)\right)\left[T^{*} M\right] .
$$

Remark 3.7. Since the images of $\operatorname{Ind}_{f, \ell^{1}(G)}(D), \mu[D]$ and $\mu_{\ell^{1}}[D]$ coincide in $K_{0}\left(C^{*}(G)\right)$, they are all equal to $\operatorname{Ind}_{f, C^{*}(G)}(D)$, by Lemma 3.3 one may replace $\mu_{\ell^{1}}[D]$ by $\operatorname{Ind}_{f, \ell^{1}(G)}(D)$ or by $\mu[D]$ in the statement of Theorem 3.6. Just as above, the definitions of $\pi_{\sharp}\left(\operatorname{Ind}_{f, \ell^{1}(G)}(D)\right)$ and $\pi_{\sharp}[D]$ involve representative idempotents $e_{i}$ in matrices over $\ell^{1}(G)$ and $\mathcal{G} \subset G$ and 
$\omega>0$ depend on the choice of $e_{0}, e_{1}$ and $P$. A similar remark applies to Ind $_{f, G}(D) \in K_{0}(\mathbb{C}[G] \otimes \mathcal{R})$ since one can pair idempotents in matrices over $\mathbb{C}[G] \otimes \mathcal{R}$ with sufficiently multiplicative quasi-representations.

Corollary 3.8. If $M^{n}$ is oriented, then by integration over the fibers of $p$ : $T M \rightarrow M$ one obtains under the assumptions and the setup of Theorem 3.6,

$$
\tau\left(\pi_{\sharp}\left(\mu_{\ell^{1}}[D]\right)=(-1)^{n(n+1) / 2}\left(p_{!} \operatorname{ch}(\sigma(D)) \cup \operatorname{Td}(T M \otimes \mathbb{C}) \cup \operatorname{ch}{ }_{\tau}\left(E_{\pi}\right)[M] .\right.\right.
$$

Let $M$ be a closed manifold with universal cover $\widetilde{M}$ and classifying map $f: M \rightarrow B G$. Let $P=P^{(M, f)}$ be a projection in matrices over $C(M) \otimes \mathbb{C}[G]$ that represents the Mishchenko line bundle $\widetilde{M} \times{ }_{G} \ell^{1}(G) \rightarrow M$ constructed as above. The corresponding "assembly" map is denoted by $\mu^{(M, f)}: K_{0}(M) \rightarrow$ $K_{0}\left(\ell^{1}(G)\right)$. If $\pi$ is a quasi-representation of $G$ we define $E_{\pi}=E_{\pi}^{(M, f)}$ as in (36). The following is a variation of a result of Hilsum and Skandalis [12], see also [10]. In this context, similar results were obtained by Hanke and Schick [11] using sequences of almost-flat bundles. Let $\mathcal{L}(M)$ denote the stable L-class of $M$.

Corollary 3.9. Let $M, N$ be two closed connected oriented manifolds and let $h: N \rightarrow M$ be an oriented homotopy equivalence. There exist a finite subset $\mathcal{G} \subset G$ and $\varepsilon>0$ such that for any $(\mathcal{G}, \varepsilon)$-unitary representation $\pi: G \rightarrow B$ into a unital $C^{*}$-algebra $B$ with a trace $\tau$, one has $\left(\mathcal{L}(N) \cup h^{*} h_{\tau}\left(E_{\pi}\right)\right)[N]=$ $\left(\mathcal{L}(M) \cup \operatorname{ch}_{\tau}\left(E_{\pi}\right)\right)[M]$.

Proof. Let $D_{M}$ be the signature operator of $M$. Then $\operatorname{Ind}_{f, C^{*}(G)}\left(D_{M}\right)$ is an oriented homotopy invariant of $M$ by results of Mishchenko and Kasparov, and hence $\operatorname{Ind}_{f, C^{*}(G)}\left(D_{M}\right)=\operatorname{Ind}_{f \circ h, C^{*}(G)}\left(D_{N}\right)$. By Lemma 3.3, $\pi_{\sharp}\left(\mu^{(M, f)}\left[D_{M}\right]\right)=\pi_{\sharp}\left(\mu^{(N, f \circ h)}\left[D_{N}\right]\right)$ if $\pi$ is sufficiently multiplicative. On the other hand, $h^{*}\left(P^{(M, f)}\right)=P^{(N, f \circ h)}$ and hence $h^{*}\left(E_{\pi}^{(M, f)}\right) \cong E_{\pi}^{(N, f \circ h)}$. We conclude the proof by applying Corollary 3.8 .

Using Theorem 3.6 in the case when $G=\pi_{1}(M)$ and $B=M_{m}(\mathbb{C})$, one can give a new proof of the following theorem from [5], see also [21]. Let $\widetilde{M}$ be the universal cover of a closed connected Riemannian manifold $M$ with classifying map $f: M \rightarrow B G$ and let $D$ be as above.

Theorem 3.10 (Connes, Gromov and Moscovici). There is $\varepsilon_{0}>0$ such that if $E$ is any $\varepsilon$-flat smooth Hermitian vector bundle on $M$ with $\varepsilon<\varepsilon_{0}$ and $\pi$ is the associated quasi-representation of $G=\pi_{1}(M)$, then

$$
\pi_{\sharp}\left(\operatorname{Ind}_{f, G}(D)\right)=(-1)^{n}(\operatorname{ch}(\sigma(D)) \cup \operatorname{Td}(T M \otimes \mathbb{C}) \cup \operatorname{ch}(E))\left[T^{*} M\right] .
$$


Proof. We only give a sketch of the proof. The argument relies on the correspondence between almost flat vector bundles on $M$ and finite dimensional quasi-representations of the fundamental group of $M$. By definition of $\varepsilon$ flatness, $E$ is a Hermitian vector bundle over $M$ which admits a metric preserving connection $\nabla$ such that if $\Theta=\nabla^{2}$ is the curvature of $\nabla$, then

$$
\|(E, \nabla)\|:=\sup \left\{\left\|\Theta_{x}(X, Y)\right\|:\|X \wedge Y\| \leq 1, X, Y \in T_{x} M, x \in M\right\}<\varepsilon .
$$

One constructs a quasi-representation $\pi: G \rightarrow L\left(\mathbb{C}^{m}\right)$ using parallel transport as in [5], where $m$ is the rank of $E$. By Lemma 4 of [5], for any finite subset $\mathcal{G} \subset G$, there is $C>0$ such that $\|\pi(s t)-\pi(s) \pi(t)\| \leq C\|(E, \nabla)\|$ for all couples $(E, \nabla)$ and $s, t \in \mathcal{G}$. Therefore, for any given $\omega>0, \pi$ will be a $(\mathcal{G}, \omega)$-unitary representation provided that $\varepsilon$ is sufficiently small. Conversely, for any given $\varepsilon>0$, there exist a finite set $\mathcal{G}$ of $G$ and $\omega>0$ such that one can associate an $\varepsilon$-flat vector bundle $E_{\pi}$ to any $(\mathcal{G}, \omega)$-unitary representation of $G$ via a construction of [23, Sect. 3, and Appendix], see also [19]. Moreover, one verifies that in agreement with our earlier notation one has in fact that $\left[E_{\pi}\right]=(1 \otimes \pi)_{\sharp}(P)$, where $P$ is the projection corre-

sponding to the $\widetilde{M} \times_{G} \ell^{1}(G) \rightarrow M$. As noted in the remark on page 312 of [25], if a $(\mathcal{G}, \omega)$-unitary representation $\pi$ is associated to an $\varepsilon$-flat bundle $E$, one can take $E$ as a particular model of $E_{\pi}\left(E_{\pi} \cong E\right)$. One concludes the proof by applying Theorem 3.6 and Remark 3.7 for the quasi-representation $\pi$ associated to $E$.

In order to derive very explicit formulas from Corollary 3.8 it would be desirable to compute $c h_{\tau}\left(E_{\pi}\right)$. In the next section we work out this computation for a Riemaniann surface of genus $n>0$ in the scalar case $B=M_{k}(\mathbb{C})$.

\section{QuASI-REPRESEnTAtions of SURFACE GROUPS}

Given two unitaries $u, v \in U(m)$ such that $\|u v-v u\|<\varepsilon$, Loring has introduced in [17] an integral invariant $\kappa(u, v)$ defined for small $\varepsilon$. One can view the pair $u, v$ as associated with a quasi-representation $\varphi: C\left(\mathbb{T}^{2}\right) \rightarrow M_{m}(\mathbb{C})$, such that $\varphi\left(z_{1}\right)=u$ and $\varphi\left(z_{2}\right)=v$, where $z_{i}$ are the canonical unitary generators of $C\left(\mathbb{T}^{2}\right)$. Then one defines $\kappa(u, v):=\varphi_{\sharp}(\beta)$, where $K_{0}\left(C\left(\mathbb{T}^{2}\right)\right) \cong$ $\mathbb{Z} \oplus \mathbb{Z} \beta$ and $\beta$ is the Bott element. On the other hand, Loring and Exel [9] introduced another invariant $\omega(u, v)$ defined as the winding number in $\mathbb{C} \backslash\{0\}$ of the loop $t \mapsto \operatorname{det}((1-t) u v+t v u)$ and they proved the equality $\kappa(u, v)=\omega(u, v)$. Exel pointed out that $\omega(u, v)=-\frac{1}{2 \pi i} \operatorname{Tr} \log \left(u v u^{-1} v^{-1}\right)$ and reproved the equality $\kappa(u, v)=\omega(u, v)$ using the soft torus $\mathrm{C}^{*}$-algebra, see $[8]$. 
In this section we use the results from Section 3 to generalize the result of Loring and Exel as follows. Two almost commuting unitaries can be viewed as corresponding to a quasi-representation of $\mathbb{Z}^{2}=\pi_{1}\left(\mathbb{T}^{2}\right)$. More generally, we are going to consider quasi-representations of surface groups, $\Gamma_{n}=\pi_{1}\left(Y_{n}\right)$, where $Y_{n}$ is a compact oriented Riemann surface of genus $n \geq 1$. It is well-known that $\Gamma_{n}$ has a standard presentation as a one-relator group

$$
\Gamma_{n}=\left\langle s_{1}, t_{1}, \ldots, s_{n}, t_{n} ; \prod_{i=1}^{n}\left[s_{i}, t_{i}\right]\right\rangle,
$$

(where $[s, t]=s t s^{-1} t^{-1}$ ) which we will review in the sequel. But first let us note the following consequence of Corollary 3.5 (or Theorem 3.6).

The assembly map $\mu_{\ell^{1}}: K_{0}\left(Y_{n}\right) \rightarrow K_{0}\left(\ell^{1}\left(\Gamma_{n}\right)\right)$ (to be denoted in the remainder of this section by $\mu$ ) is an isomorphism [16], since $\Gamma_{n}$ has the Haagerup property. Thus $K_{0}\left(\ell^{1}\left(\Gamma_{n}\right)\right) \cong \mathbb{Z} \oplus \mathbb{Z} \mu\left[Y_{n}\right]$ where $z=\left[Y_{n}\right]$ is the fundamental class of $Y_{n}$ in K-homology. It is known that $\left[Y_{n}\right]=\left[\bar{\partial}_{n}\right]+(n-$ 1)[1], where $\bar{\partial}_{n}$ is the Dolbeault operator for some (any) complex structure on $Y_{n}$ and [1] denotes the class of a character $C\left(Y_{n}\right) \rightarrow \mathbb{C}$. Moreover, the Chern character of $\left[Y_{n}\right]$ is equal to the fundamental class of $Y_{n}$ in $H_{2}\left(Y_{n}, \mathbb{Z}\right)$, which we denote again by $\left[Y_{n}\right]$, see $[2$, Lemma 7.9].

Corollary 4.1. There exist a finite set $\mathcal{G} \subset \Gamma_{n}$ and $\omega>0$ such that if $\pi: \Gamma_{n} \rightarrow U(m)$ is $(\mathcal{G}, \omega)$-representation, then $\pi_{\sharp}\left(\mu\left[Y_{n}\right]\right)=\left\langle c_{1}\left(E_{\pi}\right),\left[Y_{n}\right]\right\rangle$.

Proof. By Corollary 3.5 applied to $B=M_{m}(\mathbb{C})$ and $z=\left[Y_{n}\right]$ we have that

$$
\pi_{\sharp}\left(\mu\left[Y_{n}\right]\right)=\left\langle\left[E_{\pi}\right],\left[Y_{n}\right]\right\rangle=\left\langle\operatorname{ch}\left[E_{\pi}\right], \operatorname{ch}\left[Y_{n}\right]\right\rangle=\left\langle c_{1}\left(E_{\pi}\right),\left[Y_{n}\right]\right\rangle .
$$

Consider the free group $\mathbb{F}_{2 n}$ with generators $\hat{s}_{1}, \hat{t}_{1}, \ldots, \hat{s}_{n}, \hat{t}_{n}$ and the canonical morphism $\rho: \mathbb{F}_{2 n} \rightarrow \Gamma_{n}, \rho\left(\hat{s}_{i}\right)=s_{i}, \rho\left(\hat{t}_{i}\right)=t_{i}$. Let $\sigma: \Gamma_{n} \rightarrow \mathbb{F}_{2 n}$ be a fixed set theoretic section of $\rho$ with the property that $\sigma\left(s_{i}^{ \pm 1}\right)=\hat{s}_{i}{ }^{ \pm 1}$ and $\sigma\left(t_{i}^{ \pm 1}\right)={\hat{t_{i}}}^{ \pm 1}$. Using $\sigma$, we can associate to each representation $\widehat{\pi}: \mathbb{F}_{2 n} \rightarrow$ $U(m)$ a quasi-representation $\pi: \Gamma_{n} \rightarrow U(m)$ defined by $\pi=\widehat{\pi} \circ \sigma$.

Any set $\left\{u_{1}, v_{1}, \ldots, u_{n}, v_{n}\right\} \subset U(m)$ determines a unique representation $\widehat{\pi}: \mathbb{F}_{2 n} \rightarrow U(m)$ such that $\widehat{\pi}\left(\hat{s}_{i}\right)=u_{i}$ and $\widehat{\pi}\left(\hat{t}_{i}\right)=v_{i}, i=1, . . n$. Define $\pi: \Gamma_{n} \rightarrow U(m)$ as above, $\pi=\widehat{\pi} \circ \sigma$. It is then easy to verify that for any given finite subset $\mathcal{G}$ of $\Gamma_{n}$ and any $\omega>0$ there is $\varepsilon>0$ such that if the unitaries $\left\{u_{1}, v_{1}, \ldots, u_{n}, v_{n}\right\} \subset U(m)$ satisfy $\left\|\prod_{i=1}^{n}\left[u_{i}, v_{i}\right]-1\right\|<\varepsilon$, then $\pi$ is a $(\mathcal{G}, \omega)$-unitary representation. Let $h: \Gamma_{n} \rightarrow \Gamma_{1}$ be the homomorphism defined by $h\left(s_{1}\right)=s_{1}, h\left(t_{1}\right)=t_{1}, h\left(s_{i}\right)=1$ and $h\left(t_{i}\right)=1$ for $i \geq 2$. We denote also by $h$ the induced map $h: Y_{n} \cong B \Gamma_{n} \rightarrow Y_{1} \cong B \Gamma_{1}$. All the maps 
in the following diagram are known to be bijections.

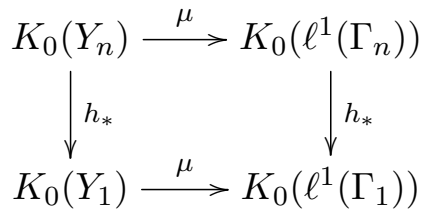

Let $\beta \in K_{0}\left(\ell^{1}\left(\Gamma_{n}\right)\right)$ be the unique element with the property that the Chern character of image of $h_{*}(\beta)$ under the canonical map $K_{0}\left(\ell^{1}\left(\Gamma_{1}\right)\right) \rightarrow$ $K_{0}\left(C^{*}\left(\Gamma_{1}\right)\right) \cong K^{0}\left(\mathbb{T}^{2}\right)$ is equal to the fundamental class $\left[\mathbb{T}^{2}\right] \in H^{2}\left(\mathbb{T}^{2}\right)$. The element $\beta$ is equal to $\mu\left[Y_{n}\right]$ up to sign, so that $K_{0}\left(\ell^{1}\left(\Gamma_{n}\right)\right) \cong \mathbb{Z} \oplus \mathbb{Z} \beta$.

We will apply Corollary 4.1 to obtain the following formula.

Theorem 4.2. There exists $\epsilon>0$ such that for any $m \geq 1$ and any set of unitaries $\left\{u_{1}, v_{1}, \ldots, u_{n}, v_{n}\right\} \subset U(m)$ with $\left\|\prod_{i=1}^{n}\left[u_{i}, v_{i}\right]-1\right\|<\varepsilon$,

$$
\pi_{\sharp}(\beta)=-\frac{1}{2 \pi i} \operatorname{Tr} \log \left(\left[u_{1}, v_{1}\right] \cdots\left[u_{n}, v_{n}\right]\right) .
$$

The proof requires some preparation. Let us view $Y_{n}$ as obtained by identifying pairs of edges in a $4 n$-gon $Q_{n}$ in the hyperbolic plane, as illustrated in Figure 1. We follow closely the presentation of this construction given in [13]. An edge of $Q_{n}$ is an oriented side of $Q_{n}$. For each edge $e$ we denote by $e^{-1}$ the result of changing the orientation. The polygon $Q_{n}$ comes with a side pairing. This is an involution $e \mapsto * e$ on the set of edges $\mathcal{E}_{n}$ of $Q_{n}$ which satisfies $*\left(e^{-1}\right)=(* e)^{-1}$. For each edge $e \in \mathcal{E}_{n}$ we denote by $\tau_{e}$ the isometry of the hyperbolic plane which maps $* e$ to $e$ in such a way that locally the half-plane bounded by $* e$ and containing $Q_{n}$ is mapped to the half-plane bounded by $e$ but opposite $Q_{n}$. One refers to $\tau_{e}$ as the transformation determined by $e$ and one has $\tau_{e^{-1}}=\tau_{e}$ and $\tau_{* e}=\tau_{e}^{-1}$. We denote by $\mathcal{E}_{n}=\left\{* a_{1}, a_{1}, * b_{1}, b_{1}, \ldots, * a_{n}, a_{n}, * b_{n}, b_{n}\right\}$ the set of edges of $Q_{n}$ oriented as shown in Figure 1 and set $\alpha_{k}=\tau_{a_{k}}, \beta_{k}=\tau_{b_{k}}$. It is shown in [13] that the group $\Gamma_{n}$ generated by these isometries is isomorphic to the one-relator group

$$
\Gamma_{n}=\left\langle\alpha_{1}, \beta_{1}, \ldots, \alpha_{n}, \beta_{n}:\left[\beta_{1}^{-1}, \alpha_{1}\right] \cdots\left[\beta_{n}^{-1}, \alpha_{n}\right]\right\rangle .
$$

Thus $s_{i}$ corresponds to $\beta_{i}^{-1}$ and $t_{i}$ corresponds to $\alpha_{i}$ from our earlier presentation of $\Gamma_{n}$.

We construct orientation preserving parametrizations for all edges as follows. First, we fix orientation preserving homeomorphisms $[0,1] \rightarrow * a_{k}$ and $[0,1] \rightarrow * b_{k}, 1 \leq k \leq n$. Then, by composing these maps with $\alpha_{k}$ and $\beta_{k}$ we obtain orientation preserving parametrizations for $a_{k}$ and $b_{k}$. It is convenient to use the same notation for a point $t \in[0,1]$ and its images $t \in * a_{k}$ and respectively $t \in * b_{k}$. 


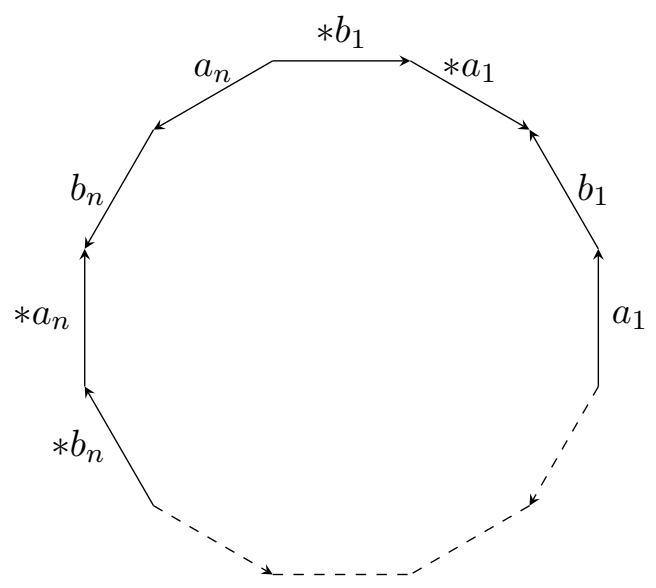

FiguRE 1. 4n-gon $Q_{n}$

Let $u_{k}, v_{k} \in U(m), k=1, \ldots, n$ with the property that

$$
\left\|\prod_{k=1}^{n}\left[v_{k}^{-1}, u_{k}\right]-1_{m}\right\|<\varepsilon<1 .
$$

Set $W_{0}=1_{m}$ and $W_{k}:=\prod_{i=1}^{k}\left[v_{i}^{-1}, u_{i}\right], \quad 1 \leq k \leq n$. We are going to construct a complex vector bundle $E$ of rank $m$ over the surface $Y_{n}$ that is a model for $E_{\pi}$. This bundle is obtained by gluing together pairs of fibers $\left(t \times \mathbb{C}^{m}\right),\left(\tau_{e} t \times \mathbb{C}^{m}\right), t \in * e$, on the edges of the base $Q_{n}$ of the trivial bundle $Q_{n} \times \mathbb{C}^{m}$ according to the following equivalence relation.

(1) $(t, \xi) \sim\left(\beta_{k} t, v_{k} \xi\right), t \in * b_{k}, \xi \in \mathbb{C}^{m}, 1 \leq k \leq n$.

(2) $(t, \xi) \sim\left(\alpha_{k} t, u_{k} \xi\right), t \in * a_{k}, \xi \in \mathbb{C}^{m}, 1 \leq k \leq n-1$.

(3) $(t, \xi) \sim\left(\alpha_{n} t, y_{n}(t) \xi\right), t \in * a_{n}, \xi \in \mathbb{C}^{m}$, where

$$
y_{n}(t)=\left(t 1_{m}+(1-t) W_{n}\right) u_{n} .
$$

Let us note also that $\left\|u_{n}-y_{n}(t)\right\|<\varepsilon$ since $\left\|1_{m}-W_{n}\right\|<\varepsilon$. One verifies immediately that $\sim$ is indeed an equivalence relation. This property would not hold for $y_{n}=u_{n}$ if $W_{n} \neq 1$. To explain why we consider the bundle $E$, let us note that the projection $P$ from Theorem 3.2 corresponds to the bundle on $Y_{n}$ obtained as the quotient of $Q_{n} \times \ell^{1}\left(\Gamma_{n}\right)$ by the equivalence relation:

$\left(1^{\prime}\right)(t, \xi) \sim\left(\beta_{k} t, \beta_{k} \xi\right), t \in * b_{k}, \xi \in \ell^{1}\left(\Gamma_{n}\right), 1 \leq k \leq n$.

(2') $(t, \xi) \sim\left(\alpha_{k} t, \alpha_{k} \xi\right), t \in * a_{k}, \xi \in \ell^{1}\left(\Gamma_{n}\right), 1 \leq k \leq n-1$.

(3') $(t, \xi) \sim\left(\alpha_{n} t, \alpha_{n} \xi\right), t \in * a_{n}, \xi \in \ell^{1}\left(\Gamma_{n}\right)$.

Using a standard perturbation argument, one verifies that if $\varepsilon$ is sufficiently small, then the projection associated to $(1 \otimes \pi)(P)$ corresponds to a vector 
bundle isomorphic to $E$ and hence $[E]=\left[E_{\pi}\right]$. Indeed, this is seen by comparing the equivalence relation defined by $(1),(2),(3)$ with the one defined by $\left(1^{\prime}\right),\left(2^{\prime}\right),\left(3^{\prime}\right)$. If $\varepsilon$ is sufficiently small, then $(1 \otimes \pi)_{\sharp}(P)=[E]$, since $\pi\left(\beta_{k}\right)=v_{k}, 1 \leq k \leq n, \pi\left(\alpha_{k}\right)=u_{k}, 1 \leq k \leq n-1$ and $\left\|\pi\left(\alpha_{n}\right)-y_{n}(t)\right\|=$ $\left\|u_{n}-y_{n}(t)\right\|<\varepsilon$. It follows that $c_{1}\left((1 \otimes \pi)_{\sharp}(P)\right)=c_{1}\left(E_{\pi}\right)=c_{1}(E)$.

We compute $c_{1}(E)$, by realizing it as the first obstruction for the existence of a cross-section in the principal $G L(m, \mathbb{C})$-bundle $F$ associated to $E$.

Proposition 4.3. The first Chern class $c_{1}(E) \in H^{2}\left(Y_{n}, \mathbb{Z}\right) \cong \mathbb{Z}$ is given (up to a sign) by the winding number of $t \mapsto \operatorname{det}\left(t 1_{m}+(1-t) W_{n}\right)$ and hence it is equal to $\pm \frac{1}{2 \pi i} \operatorname{Tr}\left(\log W_{n}\right)$.

Proof. We begin by constructing a cross-section $\Phi$ in $F$ over the 1-skeleton of $Y_{n}$. Choose continuous paths $t \mapsto V_{k}(t)$ and $t \mapsto U_{k}(t), t \in[0,1]$ in $U(m)$ such that $V_{k}(0)=U_{k}(0)=1_{m}$ and $V_{k}(1)=v_{k}, U_{k}(1)=u_{k}$. The map $\Phi$ will given by a continuous map $\phi: \mathcal{E}_{n} \rightarrow U(m)$, defined on the boundary $\mathcal{E}_{n}$ of $Q_{n}$ as follows.

For $1 \leq k \leq n$ and $t \in * b_{k}, \beta_{k} t \in b_{k}$,

$$
\phi(t)=V_{k}(t)^{-1} U_{k}(t)^{-1} V_{k}(t) W_{k-1}^{-1}
$$

and $\phi\left(\beta_{k} t\right)=v_{k} \phi(t)=V_{k}(1) V_{k}(t)^{-1} U_{k}(t)^{-1} V_{k}(t) W_{k-1}^{-1}$.

For $1 \leq k \leq n-1$ and $t \in * a_{k}, \alpha_{k} t \in a_{k}$,

$$
\phi(t)=V_{k}(t) V_{k}(1)^{-1} U_{k}(1)^{-1} V_{k}(1) W_{k-1}^{-1}
$$

and $\phi\left(\alpha_{k} t\right)=u_{k} \phi(t)=U_{k}(1) V_{k}(t) V_{k}(1)^{-1} U_{k}(1)^{-1} V_{k}(1) W_{k-1}^{-1}$.

For $t \in * a_{n}$ and $\alpha_{n} t \in a_{n}$,

$$
\begin{gathered}
\phi(t)=V_{n}(t) V_{n}(1)^{-1} U_{n}(1)^{-1} V_{n}(1) W_{n-1}^{-1}, \quad \text { and } \\
\phi\left(\alpha_{n} t\right)=y_{n}(t) \phi(t)=\left(t 1_{m}+(1-t) W_{n}\right) U_{n}(1) V_{n}(t) V_{n}(1)^{-1} U_{n}(1)^{-1} V_{n}(1) W_{n-1}^{-1} .
\end{gathered}
$$

One verifies that $\phi$ is well-defined and that $\phi\left(\beta_{k} t\right)=v_{k} \phi(t)$ for $t \in * b_{k}$, $1 \leq k \leq n, \phi\left(\alpha_{k} t\right)=u_{k} \phi(t)$ for $t \in * a_{k}, 1 \leq k \leq n-1$, and $\phi\left(\alpha_{n} t\right)=$ $y_{n}(t) \phi(t)$ for $t \in * a_{n}$. Since $\phi$ satisfies these relations, it defines a crosssection $\Phi$ in $F$ over the 1-skeleton of $Y_{n}$. The first Chern class of $E$ (or $F$ ) is represented by the obstruction to extending $\phi$ from the boundary of $Q_{n}$ to $Q_{n}$. This obstruction lies in $H^{2}\left(Y_{n}, \pi_{1}(G L(m, \mathbb{C})) \cong \pi_{1}(G L(m, \mathbb{C})) \cong \mathbb{Z}\right.$. The map det $: G L(m, \mathbb{C}) \rightarrow \mathbb{C} \backslash\{0\}$ induces an isomorphism of fundamental groups. The restriction of $\operatorname{det} \phi(-)$ to four successive edges $* b_{k}, * a_{k}, b_{k}^{-1}, a_{k}^{-1}$, $1 \leq k \leq n-1$, gives a loop in $\mathbb{C} \backslash\{0\}$ based at 1 . This loop is obtained by the concatenation of the paths $\operatorname{det}\left(U_{k}(t)^{-1}\right), \operatorname{det}\left(V_{k}(t) U_{k}(1)^{-1}\right)$, 
$\operatorname{det}\left(V_{k}(1) U_{k}(1-t)^{-1}\right)$ and $\operatorname{det}\left(V_{k}(1-t)\right)$. We note that this loop is trivial in $\pi_{1}(\mathbb{C} \backslash\{0\}, 1)$ via the homotopy of loops which for $s \in[0,1]$ is obtained by the concatenation of the paths $\operatorname{det}\left(U_{k}(s t)^{-1}\right), \operatorname{det}\left(V_{k}(s t) U_{k}(s)^{-1}\right)$, $\operatorname{det}\left(V_{k}(s) U_{k}(s(1-t))^{-1}\right)$ and $\operatorname{det}\left(V_{k}(s(1-t))\right)$ respectively. By a similar reasoning, the restriction of $\operatorname{det} \phi(-)$ to the edges $* b_{n}, * a_{n}, b_{n}^{-1}, a_{n}^{-1}$ is homotopic to $t \mapsto \operatorname{det}\left((1-t) 1_{m}+t W_{n}\right)$. This shows that $c_{1}(E)=c_{1}(\operatorname{det}(E))$ is given (up to a sign) by the winding number of $t \mapsto \operatorname{det}\left(t 1_{m}+(1-t) W_{n}\right)$. Finally, it is known and immediate that there is $\theta>0$ with the following property. Let $w \in G L(m, \mathbb{C})$ be such that $\|w-1\|<\theta$ and $\operatorname{det}(w)=1$. The winding number of $t \mapsto \operatorname{det}\left((1-t) 1_{m}+t w\right)$ is equal to $\frac{1}{2 \pi i} \operatorname{Tr}(\log w)$, where $\log$ is the branch of the natural $\operatorname{logarithm}$ with $\log 1=0$.

Conclusion of proof of Theorem 4.2. Since we have seen that $\left[E_{\pi}\right]=[E]$ the result follows (up to a sign) from Corollary 4.1 and Proposition 4.3. In order to determine the appropriate sign it suffices to consider the case of a quasi-representation $\pi: \Gamma_{n} \rightarrow U(m)$ of the form $\pi=\pi^{\prime} \circ h$, where $h: \Gamma_{n} \rightarrow \Gamma_{1}$ is the morphism and $\pi^{\prime}$ is a quasi-representation of $\Gamma_{1}$ described earlier, corresponding to Voiculescu's unitaries [27]. In other words it suffices to determine the sign in the case of the 2-torus. From the calculations done in [17] for Voiculescu's unitaries, one concludes that $\pi_{\sharp}(\beta)=$ $-\frac{1}{2 \pi i} \operatorname{Tr} \log \left(u v u^{-1} v^{-1}\right)$.

Remark 4.4. Another possible approach to the computation of $c_{1}\left(E_{\pi}\right)$ is to appeal to a formula from a survey paper of Manuilov and Mishchenko [19]. That entails to working with a triangularization of $Y_{n}$ and becomes quite laborious. For instance, the minimum number of triangles in a triangulation of the torus is 14. Manuilov [18] has results on homotopy properties of quasirepresentations of group surfaces that can be used to deduce Theorem 4.2 from the original Exel-Loring formula.

\section{REFERENCES}

[1] M. F. Atiyah and I. M. Singer. The index of elliptic operators. III. Ann. of Math. (2), 87:546-604, 1968.

[2] H. Bettaieb, M. Matthey, and A. Valette. Unbounded symmetric operators in $K$ homology and the Baum-Connes conjecture. J. Funct. Anal., 229(1):184-237, 2005.

[3] B. Blackadar. K-theory for operator algebras, volume 5 of Mathematical Sciences Research Institute Publications. Cambridge University Press, Cambridge, second edition, 1998.

[4] A. Connes. Noncommutative Geometry. Academic Press, San Diego, CA, 1994.

[5] A. Connes, M. Gromov, and H. Moscovici. Conjecture de Novikov et fibrés presque plats. C. R. Acad. Sci. Paris Sér. I Math., 310(5):273-277, 1990. 
[6] A. Connes and H. Moscovici. Cyclic cohomology, the Novikov conjecture and hyperbolic groups. Topology, 29(3):345-388, 1990.

[7] M. Dadarlat. Group quasi-representations that act nontrivially on K-theory and almost flat bundles. preprint 2011.

[8] R. Exel. The soft torus and applications to almost commuting matrices. Pacific J. Math., 160(2):207-217, 1993.

[9] R. Exel and T. A. Loring. Invariants of almost commuting unitaries. J. Funct. Anal., 95(2):364-376, 1991.

[10] B. Hanke and T. Schick. Enlargeability and index theory. J. Differential Geom., 74(2):293-320, 2006.

[11] B. Hanke and T. Schick. Enlargeability and index theory:infinite covers. K-Theory, 38(1):23-33, 2007.

[12] Hilsum-Skandalis. Invariance par homotopie de la signature à coefficients dans un fibré presque plat. J. Reine Angew. Math., 423:73-99, 1992.

[13] B. Iversen. Hyperbolic geometry, volume 25 of London Mathematical Society Student Texts. Cambridge University Press, Cambridge, 1992.

[14] G. G. Kasparov. Equivariant $K K$-theory and the Novikov conjecture. Invent. Math., 91(1):147-201, 1988.

[15] V. Lafforgue. Banach $K K$-theory and the Baum-Connes conjecture. In Proceedings of the International Congress of Mathematicians, Vol. II (Beijing, 2002), pages 795812, Beijing, 2002. Higher Ed. Press.

[16] V. Lafforgue. $K$-théorie bivariante pour les algèbres de Banach et conjecture de BaumConnes. Invent. Math., 149(1):1-95, 2002.

[17] T. A. Loring. K-theory and asymptotically commuting matrices. Canad. J. Math., 40(1):197-216, 1988.

[18] V. M. Manuilov. Almost-representations and asymptotic representations of discrete groups. Izv. Akad. Nauk SSSR Ser. Mat., 63(5):9951014, 1999.

[19] V. M. Manuilov and A. S. Mishchenko. Almost, asymptotic and Fredholm representations of discrete groups. Acta Appl. Math., 68(1-3):159-210, 2001. Noncommutative geometry and operator $K$-theory.

[20] A. S. Mishchenko and A. T. Fomenko. The index of elliptic operators over $C^{*}$-algebras. Izv. Akad. Nauk SSSR Ser. Mat., 43(4):831-859, 967, 1979.

[21] H. Moscovici. Cyclic cohomology and invariants of multiply connected manifolds. In Proceedings of the International Congress of Mathematicians, Vol. I, II (Kyoto, 1990), pages 675-688, Tokyo, 1991. Math. Soc. Japan.

[22] W. Paravacini. A W.L.O.G. concerning Banach pairs. (unpublished note).

[23] A. V. Phillips and D. A. Stone. The computation of characteristic classes of lattice gauge fields. Comm. Math. Phys., 131(2):255-282, 1990.

[24] T. Schick. $L^{2}$-index theorems, KK-theory, and connections. New York J. Math., 11:387-443 (electronic), 2005.

[25] G. Skandalis. Approche de la conjecture de Novikov par la cohomologie cyclique (d'après A. Connes, M. Gromov et H. Moscovici). Astérisque, (201-203):Exp. No. 739, 299-320 (1992), 1991. Séminaire Bourbaki, Vol. 1990/91.

[26] J.-L. Tu. La conjecture de Baum-Connes pour les feuilletages moyennables. K-Theory, 17(3):215-264, 1999. 
[27] D. Voiculescu. Asymptotically commuting finite rank unitary operators without commuting approximants. Acta Sci. Math. (Szeged), 45(1-4):429-431, 1983.

Department of Mathematics, Purdue University, West Lafayette IN 47907, U.S.A.

E-mail address: mdd@math.purdue.edu 